\title{
THE INTERPRETATION OF SPECTRA, POLARIZATION, AND DIRECTIVITY OF SOLAR HARD X-RAYS
}

\author{
JOHN C. BROWN \\ Space Research Laboratory of the Astronomical Institute, Utrecht, The Netherlands*
}

\begin{abstract}
The importance of interpretation of hard X-ray burst spectra, polarisation and directivity to the flare process as a whole is emphasised. After critically reviewing observations of these and related burst characteristics, the problems of analytic and numerical inversion of the X-ray spectrum to give the flare electron spectrum are discussed and it is concluded that electron spectra cannot be accurately and unambiguously inferred from their bremsstrahlung emission. Consideration of directional, albedo, and model-dependent effects, on the other hand, shows that none of the X-ray data are at present inconsistent with a power-law electron acceleration spectrum.

Characteristics of thick-target, thin-target and electron-trap models of hard X-ray sources are discussed quantitatively and their ability to fit the observations is examined. Selection of a satisfactory model is precluded by lack of both sufficient observations and of adequate theoretical description of models. Nevertheless, it is suggested that redistribution of the flaring atmosphere and the effects of collective energy losses may reconcile even behind-the-limb burst observations and interplanetary electron spectra with a thick-target description (which fits other data well). This is attractive since a thick-target X-ray source makes the minimal demand on flare energy. Even a thick-target, however, requires an embarrassingly large number and energy of fast electrons. Therefore the review is completed by discussing how these requirements might be reduced if thermal emission extended to hard $\mathrm{X}$-ray energies or if multiple reacceleration of electrons occurred.
\end{abstract}

\section{Introduction}

Interesting as some problems of the hard X-ray flare may be per se, their basic importance lies in their relationship to the fascinating and long-standing conundrum of the solar flare as a whole, reviews of which include those by Sturrock and Coppi (1966) and by Sweet $(1969,1971)$. Of the several basic requirements, listed in these reviews, for any flare model, two of the most demanding are the sufficiently rapid conversion of energy from preflare (magnetic) storage into both energetic particle streams and heating of the thermal flare plasma to produce thermal radiation and mass motions. The importance of hard X-ray bursts in this connection is, firstly, to give the most direct view of accelerated flare particles in situ, radio burst and interplanetary particle observations being relatively difficult to interpret due to propagation effects. Secondly the electrons responsible for the bursts lie at the low energy end of the steep spectrum of accelerated particles and so comprise by far the bulk of the total number and energy of these particles (Neupert, 1968; Brown, 1971, and many subsequent authors). Furthermore, there is considerable evidence that this total energy may in some flares equal or exceed the total requirements for the thermal flare phenomena (e.g. Neupert, 1968; Brown, 1971, 1972a; Kahler and Kreplin, 1971; Syrovatskii and Shmeleva, 1972; McKenzie et al., 1973).

Consequently there has been wide interest lately in the detailed development of flare

* On leave from: Dept. of Astronomy, University of Glasgow, Scotland. 
models in which the thermal flare plasma is heated by the energetic electrons responsible for the hard X-ray burst (e.g. Strauss and Papaggianis, 1971; Cheng, 1972; Hudson, 1972; Brown, 1973a; Shmeleva and Syrovatskii, 1973). Empirical evidence for this hypothesis in terms of the interrelationships of hard X-rays with thermal flare characteristics has been considered by many authors (see, e.g., reviews by Hudson (1973) and Brown (1973b)). Not only does this model result in some conceptual simplification of the overall flare problem, by linking the processes of particle acceleration and flare heating, but it also permits detailed quantitative prediction of the heated atmospheric structure (Brown, 1973a) and so testing of the model by calculation of the expected optical line profiles (Canfield, 1974).

Clearly then it is essential to have the most reliable procedure possible for inferring flare electron numbers and spectra. Uncertainties in the inference of these from X-ray burst data stem from several sources:

(1) approximations used in the conversion from X-ray to effective electron spectra in the source, both in the basic physics (e.g. bremsstrahlung cross section) and in the mathematical procedure followed;

(2) model dependence of the flux and spectrum of electrons at acceleration as against their effective values in the source;

(3) major gaps in our knowledge of the basic physical processes governing the source electrons, and especially their energy losses.

After reviewing the basic observational features of bursts, I have aimed at discussing the most important of each of these sources of uncertainty, posing the problems with which the current state of the art of burst interpretation leaves us, and suggesting some areas for investigation which might help us emerge from these.

\section{The Mechanism of Burst Emission}

It is now widely accepted that the source mechanism in the $\mathrm{keV}-\mathrm{MeV}$ range is collisional bremsstrahlung of energetic flare electrons on protons (and on heavy ions) in the flare plasma, synchrotron radiation and inverse Compton scattering having been ruled out by arguments such as Korchak's (1967a, 1971). Virtually nothing is as yet known of the role of collective interaction of the electron streams with the flare plasma in hard X-ray sources (cf. Sections V(a) and VI(c)), but it would appear that these interactions are incapable of direct generation of X-rays, due to the long wavelengths of plasmons, though they may play a vital part in the overall energy balance of the source (cf. Section VI(c)) - q.v. Tsytovich (1973) and references therein.

The source being optically thin, the total bremsstrahlung intensity may be written:

$$
I(\varepsilon, t)=\frac{1}{4 \pi R^{2}} \int_{V} n(\mathbf{r}, t) \int_{\varepsilon}^{\infty} F(E, \mathbf{r}, t) Q(\varepsilon, E) \mathrm{d} E \mathrm{~d} V,
$$

where $I(\varepsilon, t)=$ mean instantaneous photon flux from whole source at $R=1$ AU, differential in photon energy $\varepsilon ; F(E, \mathbf{r}, t)=$ instantaneous electron number flux, differential 
in electron energy $E$, at position $\mathbf{r}$ in the total (instantaneous) source volume $V$; $n(\mathbf{r}, t)=$ total proton number density at $\mathbf{r} ; Q(\varepsilon, E)=$ bremsstrahlung emission crosssection at $\varepsilon, E$, differential in $\varepsilon ; Q$ should be written to include the substantial correction factor for heavy ions (Elwert and Haug, 1971); a correction may also be made for electron-electron bremsstrahlung though this is very minor except at relativistic photon energies (Takakura, 1969).

Source Equation (1) describes bursts observed with spectral and temporal resolution but without spatial resolution and averaged over all planes of polarisation and directions of observation. Generalisations to include these latter features will be implied where necessary in the text, details being available in the references.

In addition it must be emphasised that (1) refers only to the primary emission from the source and does not include the important contribution to the total observed burst from primary photons backscattered from the photosphere. The importance of this component, first pointed out by Tomblin (1972) and by Santangelo et al. (1973), has not yet been fully realized or investigated. From the theoretical viewpoint, it requires a quite distinct treatment from the primary component since it involves transfer of photons by multiple Compton scattering and hence modification of the intensity, spectral, directional, polarisation and spatial characteristics of the total emission. The influence of these changes on the considerations in this review are mentioned in the text in so far as they have currently been worked out (Tomblin, 1972; Santangelo et al., 1973; Beigman and Vainstein, 1974; Brown et al., 1974).

\section{Resume of Observed Burst Characteristics}

\section{(a) TIME PROFILES}

Kane (1969) and Kane and Anderson (1970) have described small events in terms of impulsive and gradual components, the former having a profile consisting of a spike (sometimes repeated) of $e$-folding rise and fall times $\lesssim 10 \mathrm{~s}$, and the latter a smooth rise and fall over several minutes (Figure 1). Dominance of the impulsive over the gradual component with increasing photon energy, and the relationship to radio bursts, probably identifies the former as non-thermal bremsstrahlung of fast electrons and the latter as gradual thermal flare emission.

Larger events with total durations of minutes to tens of minutes show a complex spiky time structure, sometimes of quasi-periodic form - Figure 2 (Frost, 1969; Frost and Dennis, 1971; Parks and Winckler, 1969; Van Beek et al., 1974; Hoyng et al., 1975). Additionally, Frost and Dennis (1971) and Frost (1974a) have reported a distinct second component in some large events, after the 'impulsive' phase and with a persistent hard spectrum (Figure 3), possibly associated with a second phase of particle acceleration (Frost and Dennis, 1971).

Comparitively little has yet been achieved in the quantitative time series analysis of burst time profiles, as against phenomenological modelling. Hoyng et al. (1975), in the first detailed study of this type, have, however, shown how much more information may be extracted from existing data than is generally done. In particular, they empha- 


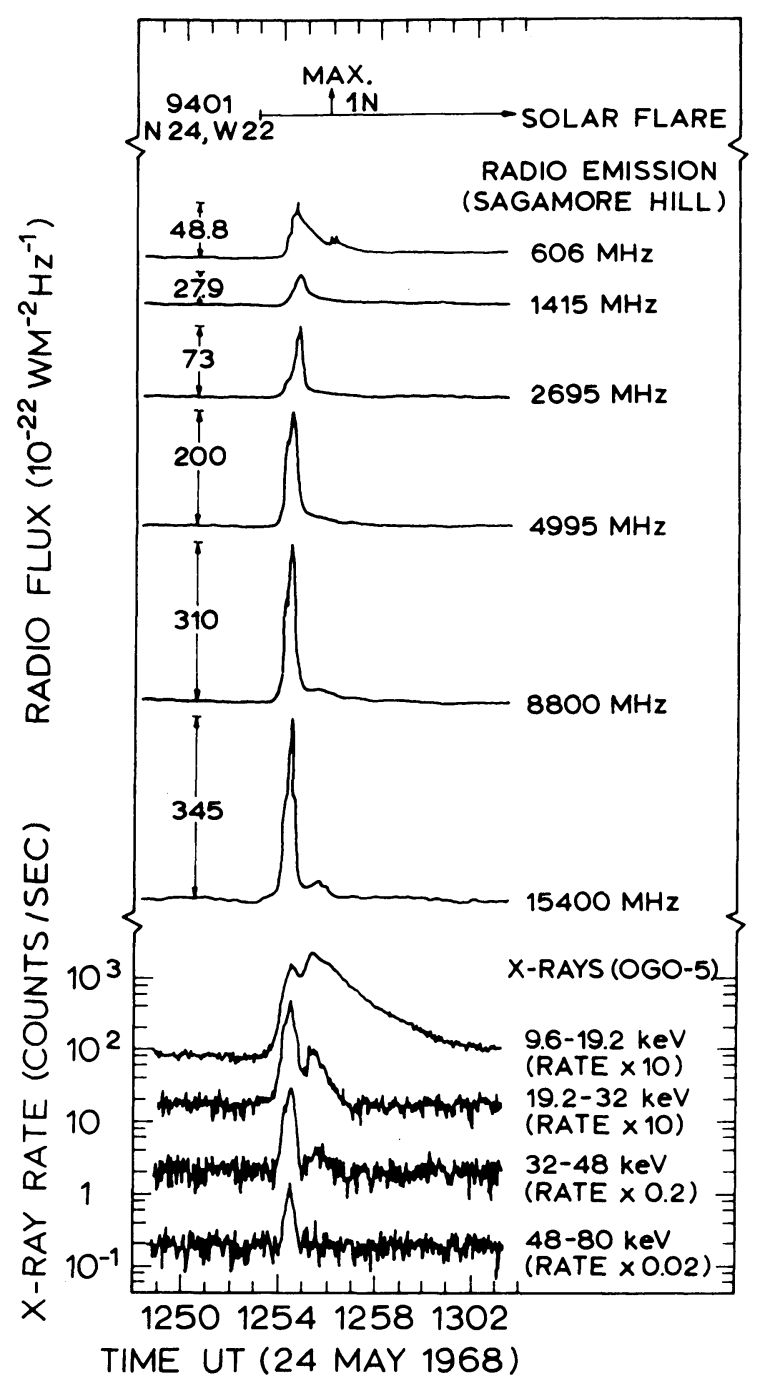

Fig. 1. An example of the simple two component (impulsive and gradual) structure of some small events reported by Kane and Anderson (1970).

sise the need for caution in the inference of time scales characterising the source from time profiles and find $e$-folding times for the source as a whole, considerably longer than their instrumental resolution (1.2 s).

(b) SPECTRAL CHARACTERISTICS

Flare $\mathrm{X}$-ray spectra above about $10 \mathrm{keV}$ are much harder than the exponential form $(1 / \varepsilon) e^{-\varepsilon / k T}$ from any isothermal plasma, a convenient and widely used representation of such spectra in astrophysics being the negative power-law $\varepsilon^{-\gamma}$. Though the real 
physical significance of this functional form is, as discussed later, very debatable, the representation is convenient for intercomparison of bursts, folding through instrumental responses, and for reasonable estimates of flare electron parameters in most events (cf. Sections IV and V).

For small events, a good spectral fit is often only obtained near the burst peak intensity. Kane (1971) has studied the statistics of this $\gamma$ for many small events and finds a frequency distribution increasing from $\gamma \gtrsim 2$ up to $\gamma \gtrsim 6$ (above $\gamma=6$, pulse

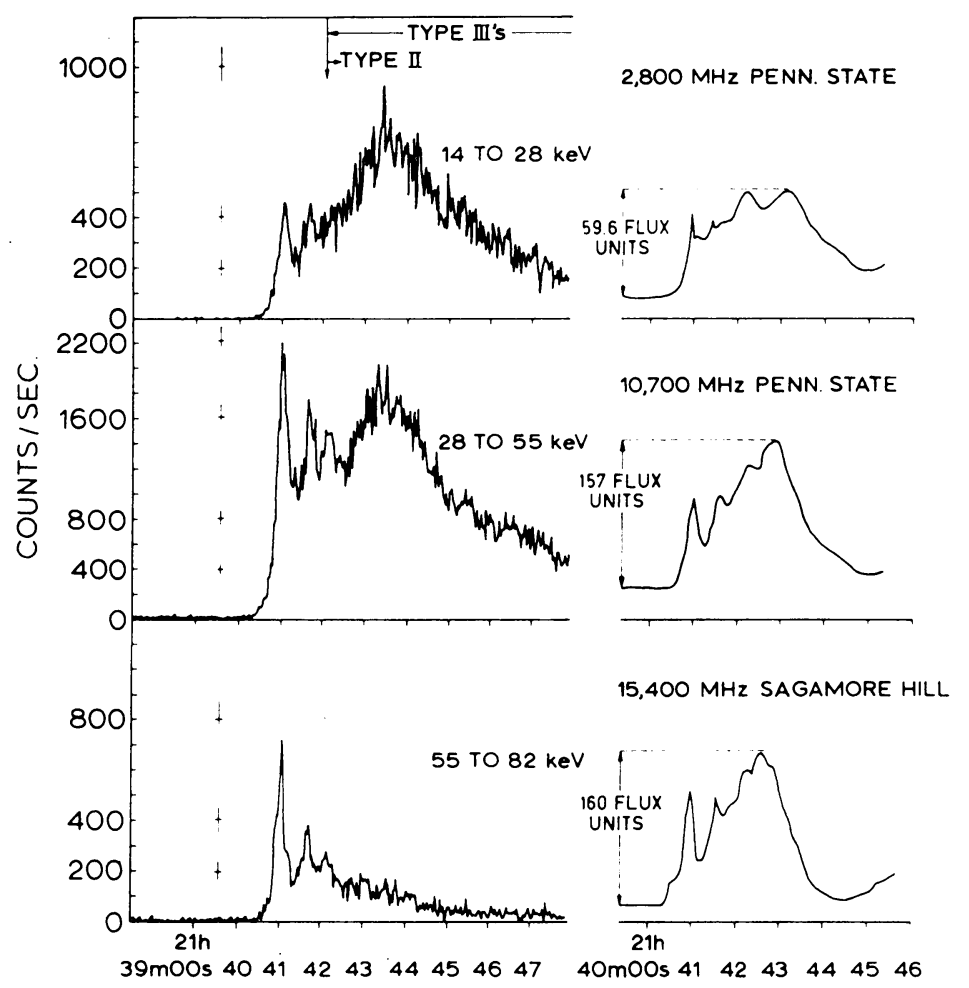

Fig. 2. Complex time structure of a large event observed by Frost (1969).

pile-up prevents determination of the true $\gamma$ ). Similar studies have been reported by Datlowe (1975). Kane (e.g. 1974a) has also investigated the correlation of X-ray burst intensities and spectra with other flare characteristics. For larger 'impulsive' events a wide range of $\gamma$ values up to over 7 has likewise been reported (e.g. Hoyng et al., 1975) while Frost's second phase apparently always has a hard spectrum $(\gamma \simeq 3)$.

Time development of spectra through events has also been studied (cf. Kane's review, 1974a). Kane and Anderson (1970) have reported that the impulsive spikes of some small events harden as they rise ( $\gamma$ decreasing) and soften as they fall ( $\gamma$ increasing) - Figure 4. Though similar behaviour has been reported for some other events (e.g. Parks and Winckler, 1969; McKenzie et al., 1973), it does not in fact seem to be 


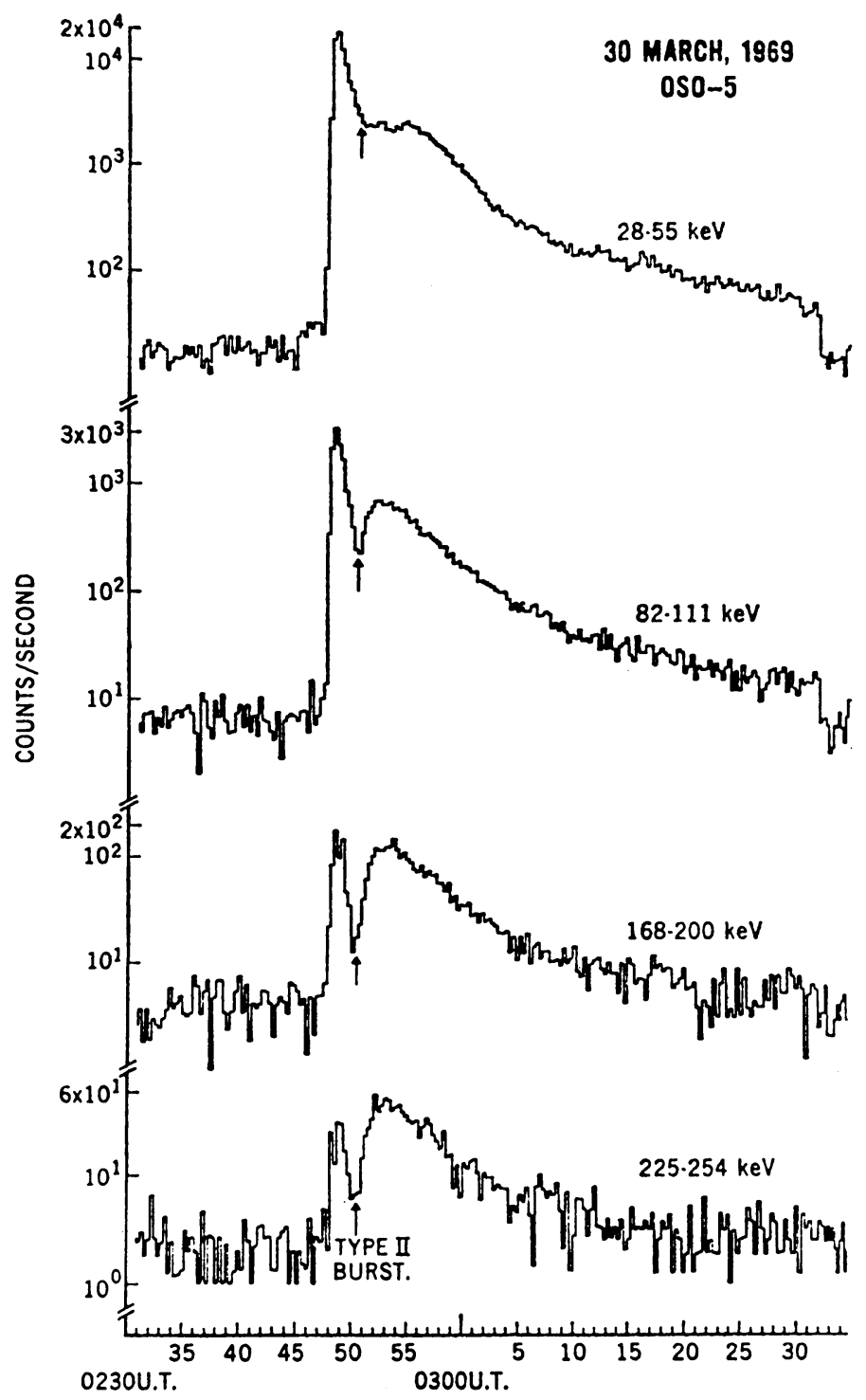

Fig. 3. Slow late component of a large burst, dominant at high energies, reported by Frost and Dennis (1971) as indicating a second stage of particle acceleration.

a general trend. In particular, Hoyng et al. (1975) have carried out the most detailed analysis available of the evolution of burst intensities and spectra, with $1.2 \mathrm{~s}$ time resolution. For none of the events studied was $\gamma$ found to evolve similarly to the results of Kane and Anderson but rather, for example, showed an increase throughout one event (Figure 5). Hoyng et al. (1975) found, however, that in the long enduring event of 1972, August $4 \gamma$ followed a systematic variation related to the burst intensity, 

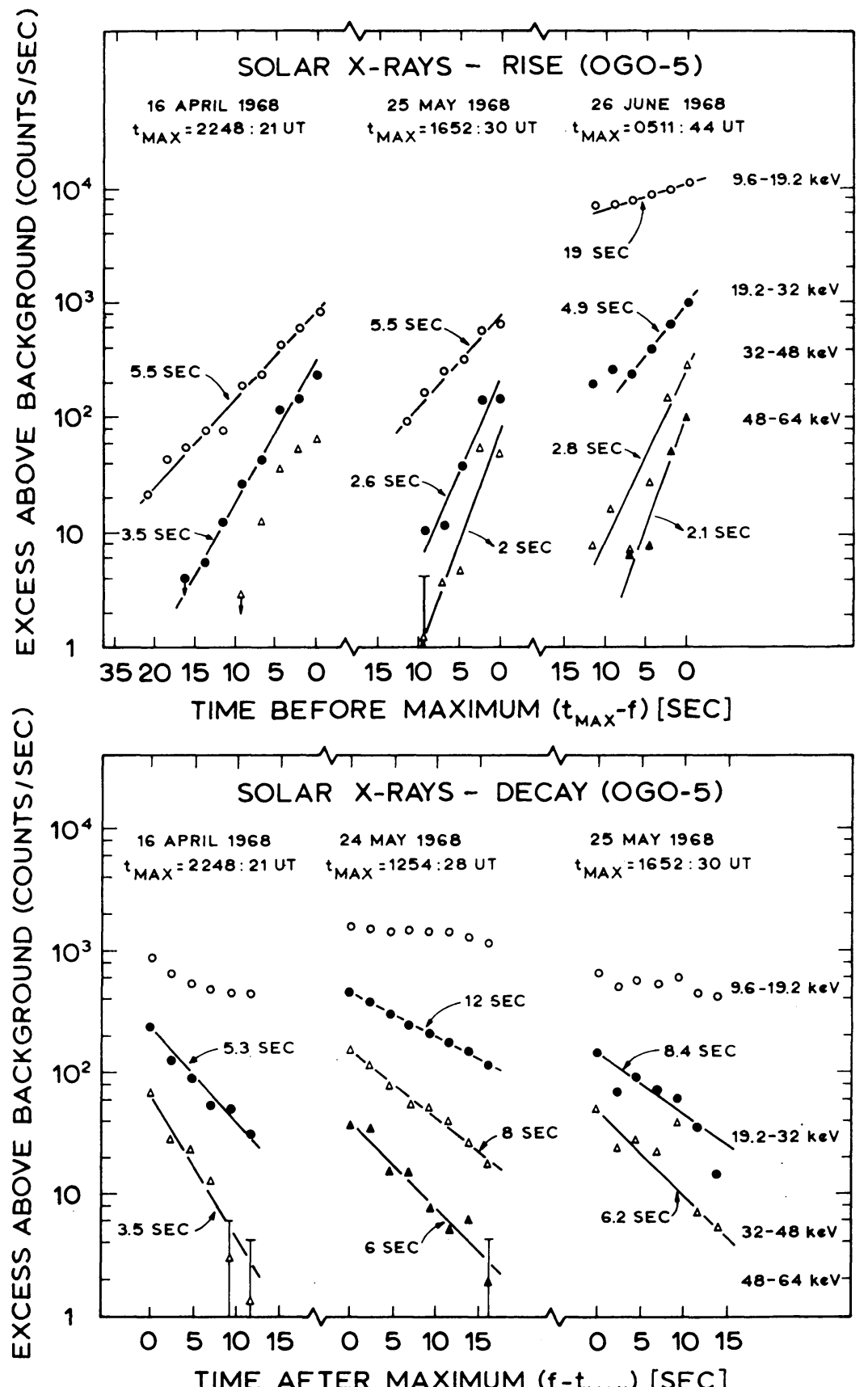

Fig. 4. Rise and decay times of small impulsive bursts as a function of photon energy (from Kane and Anderson, 1970). 


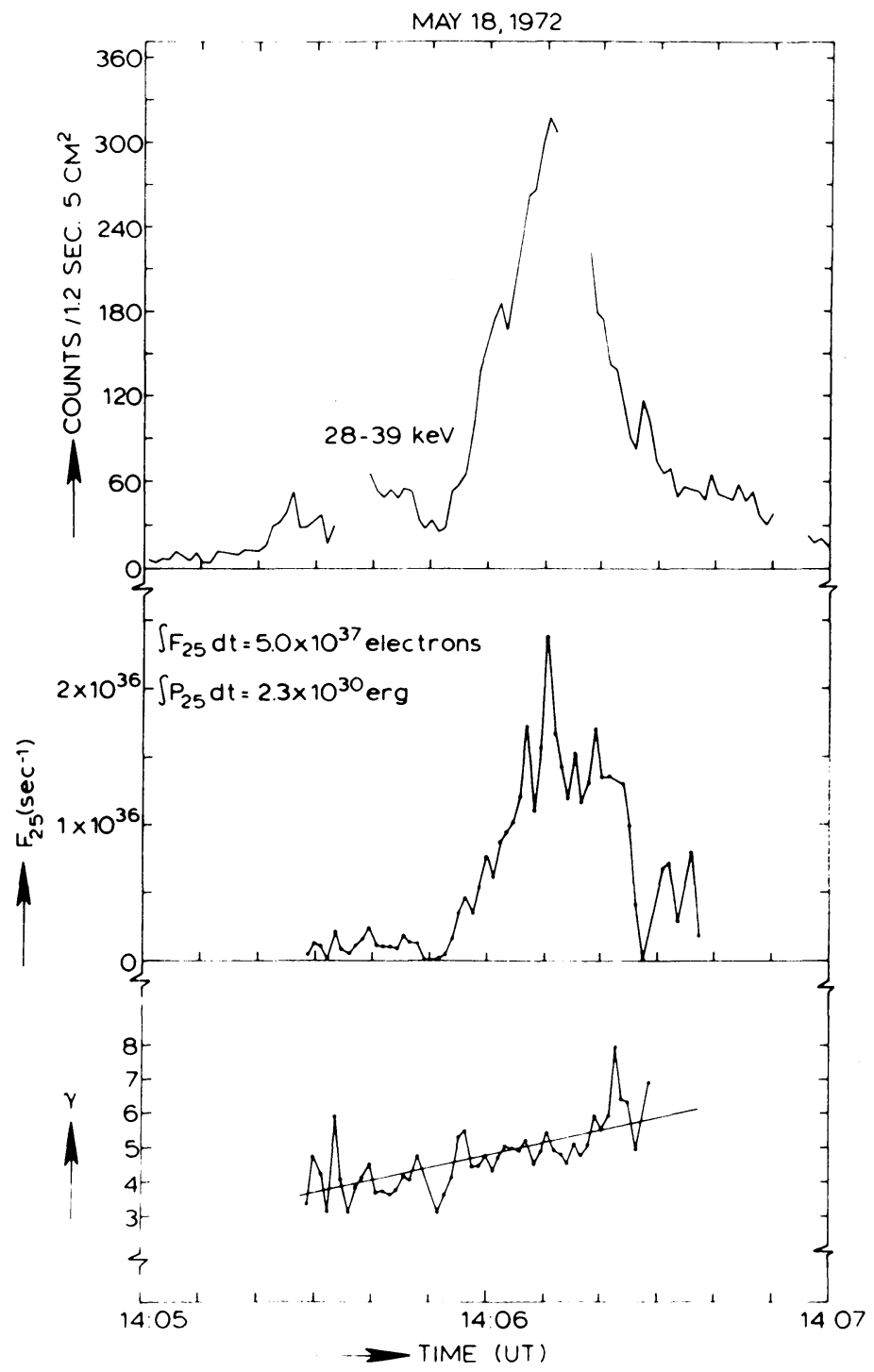

Fig. 5. Detailed time evolution of spectral index $\gamma$ in the event of May 18, 1972 (Hoyng et al., 1975). Also shown are the raw count rate and inferred thick-target electron flux $F_{25}\left(\mathrm{~s}^{-1}\right)$.

the form of this relationship changing between the rising, central, and decay portions of the time profile (Figure 6) and showing that the electron flux and spectral index are determined by some single physical parameter at each stage of this event.

It is important to resolve the discrepancy between the trend in $\gamma$ reported by Kane and Anderson (1970) and that presented by other authors since much theoretical discussion of models has been based on the observation (e.g. Kane and Anderson, 1970; Brown, 1972a; Petrosian, 1973). In particular, it is not clear whether the discrepancy 
represents a true distinction between the spectral characteristics of small and large events or whether the temporal behaviour of $\gamma$ in small events is not revealed at all by available time resolution, the apparent trend being barely extractable from the background and the rising gradual component (Takakura 1969) - cf. Figures 1 and 4.

Deviations of the burst spectrum from the power-law approximation are also important. In particular, the low energy end of the spectrum must break from a powerlaw at some point (otherwise the X-ray flux would be divergent at small $\varepsilon$ ). In practice

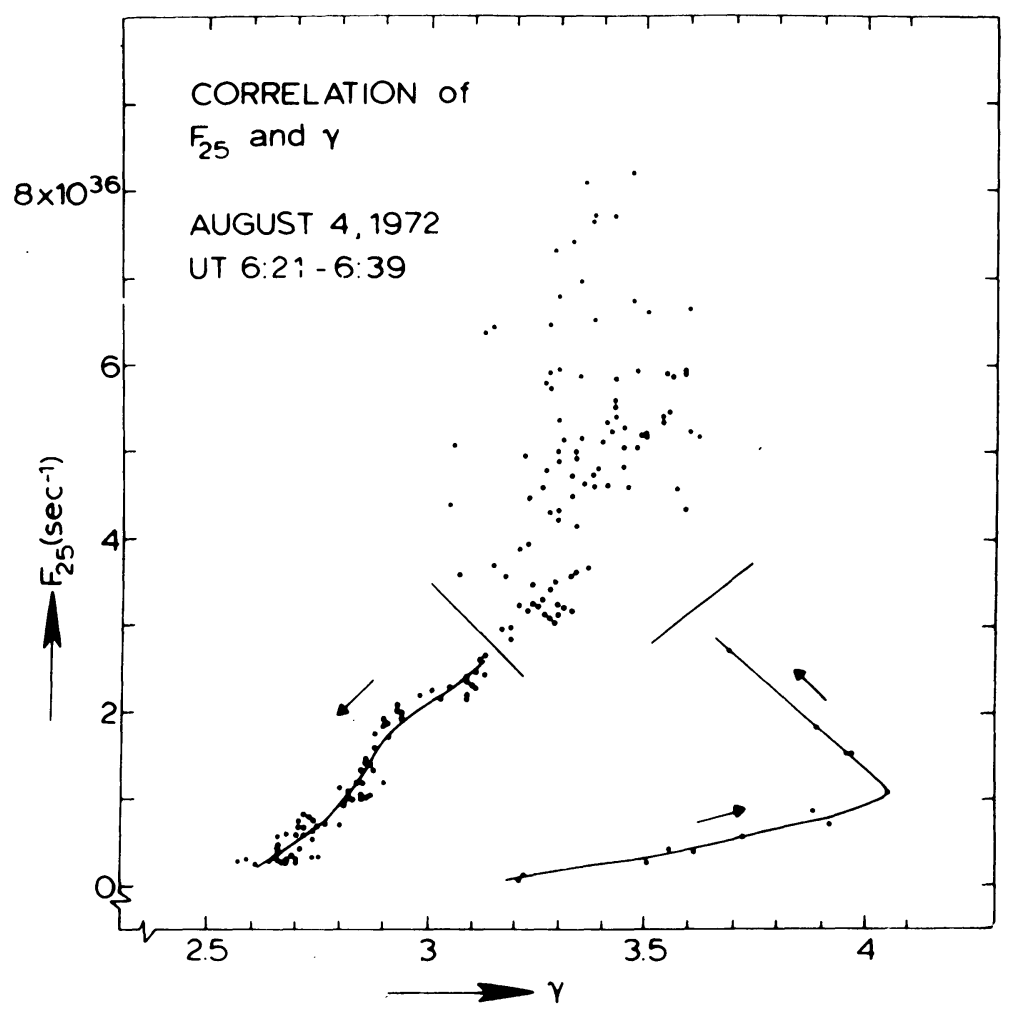

Fig. 6. Relationship of spectral index $\gamma$ to inferred electron flux $F_{25}$ through the large event of 1972, August 4 (Hoyng et al., 1975). Note the change in form of this relationship between rising and decaying phases.

the break is not directly observable since it occurs in the energy range where the gradual thermal component is dominant. Peterson et al. (1973) have however reported a powerlaw spectrum extending down as far as $6 \mathrm{keV}$ (Figure 7) while Kahler and Kreplin (1971) have attempted to infer the downward extension of the power-law from the lowest photon energy at which the impulsive spike becomes visible above the gradual component (Figure 8), namely again a few keV in some bursts. The problem is particularly important since it is the low energy cut-off in the flare electron spectrum which governs the total electron energy inferred from X-ray bursts (Neupert, 1968; Brown, 

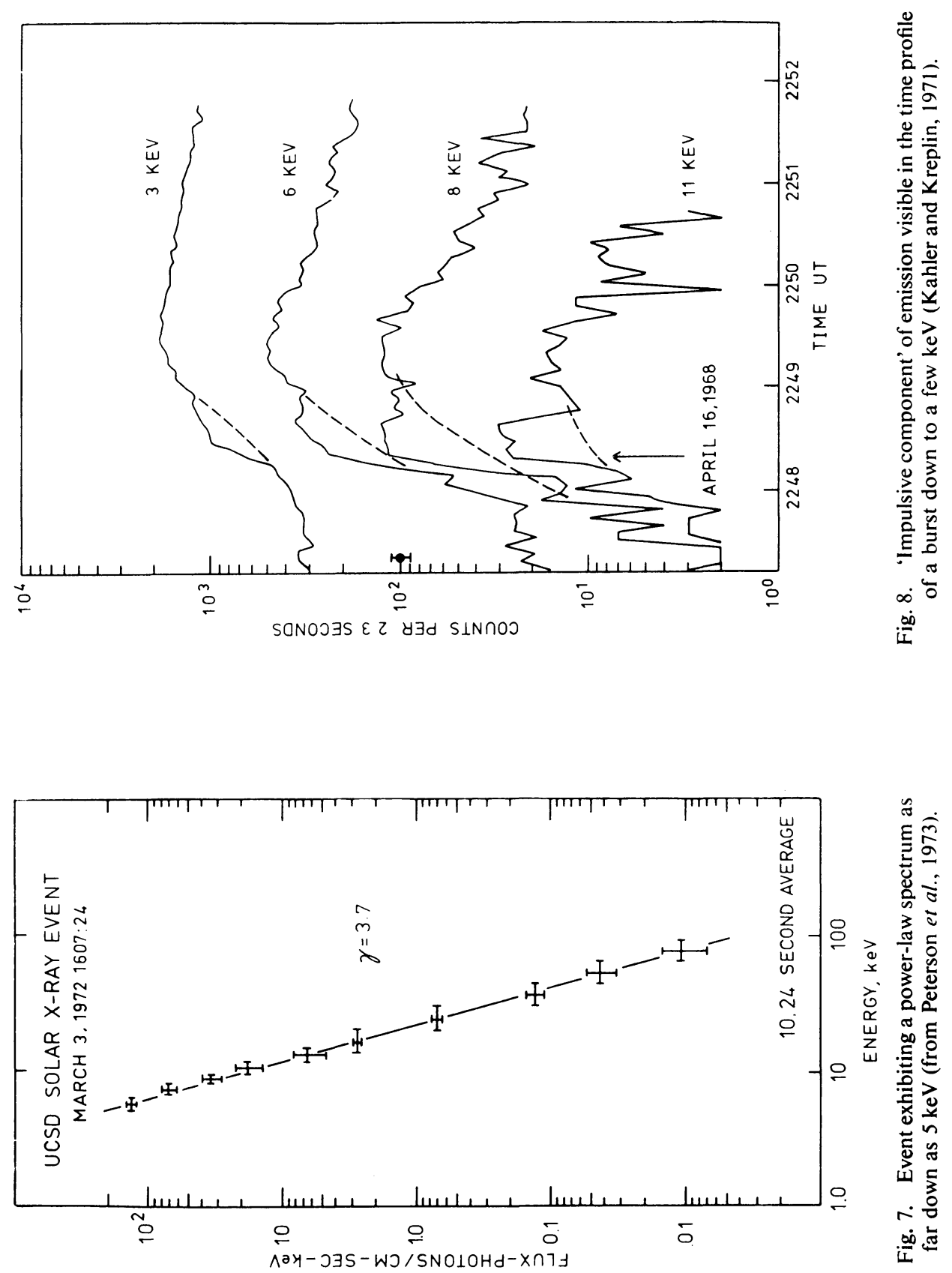

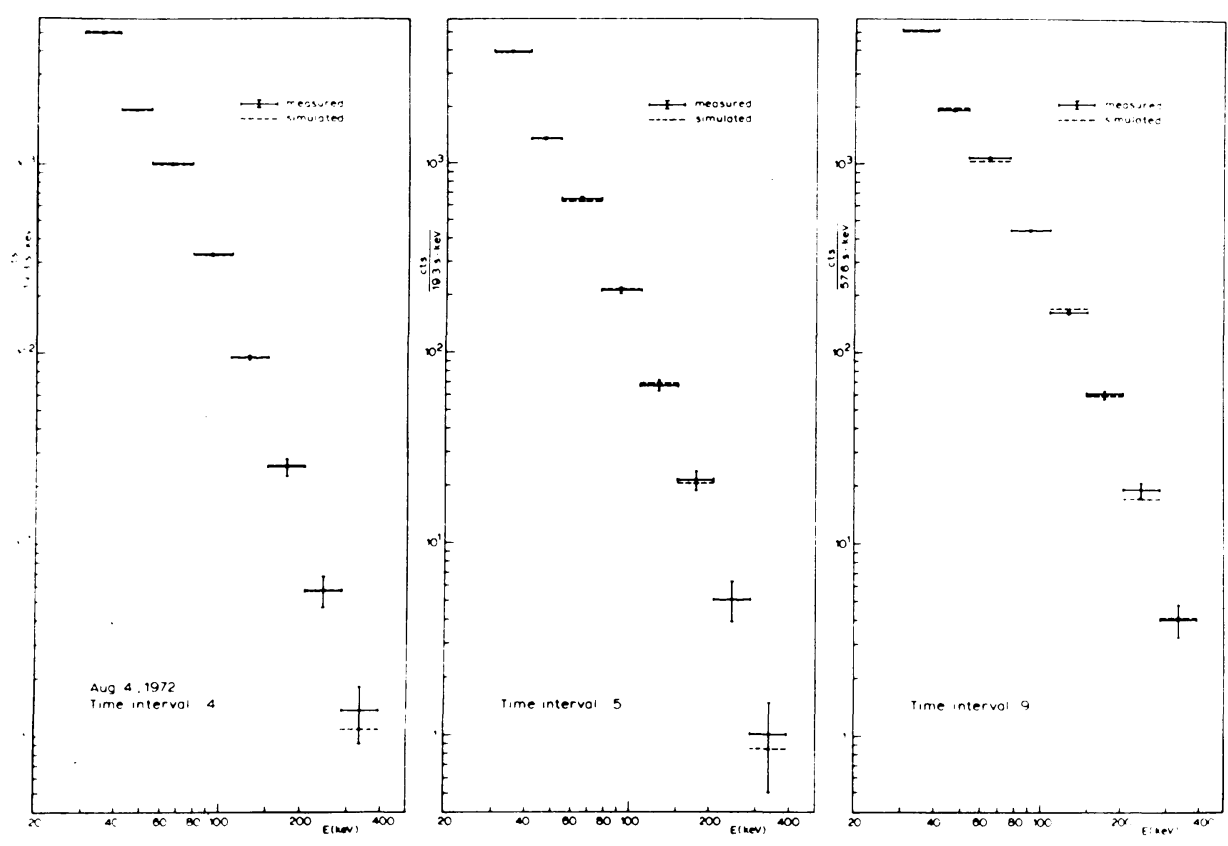

Fig. 9. High energy bend ('knee') in the photon spectrum of the burst of 1972, August 4 (from van Beek, 1973) in three different time intervals.

1971, and many subsequent authors) and so the importance attributed to the electrons in the flare as a whole (Section VI).

Steepening of the spectrum at high energies has been widely reported in both small and large events (Cline et al., 1969; Frost, 1969; Kane and Anderson, 1970; Frost and Dennis, 1971; Van Beek et al., 1974), the bend occurring generally in the 60-100 keV range (Figure 9) but sometimes as high as $500 \mathrm{keV}$ (Cline et al., 1969) and involving a change of up to 2 in $\gamma$. The location and form of this spectral 'knee' and its evolution have been considered by Van Beek et al. (1974). Its significance for the electron spectrum is not yet known (or even fully proved - cf. Section IV(e)), though as Frost (1969) has suggested it may indicate a real high energy cut-off in the acceleration process.

\section{(c) POLARISATION AND DIRECTIVITY}

The potential importance of $\mathrm{X}$-ray polarisation as a diagnostic of energetic electron motions in flares has been expounded by Elwert (1968), Elwert and Haug (1970, 1971), Haug (1972), Korchak (1967b, 1971, 1974) and Brown (1972b) while the basic methods and problems of polarisation measurement in practice have been considered by Wolff (1973) and by Thomas (1975). Only Tindo et al. (1970, 1972a, b, 1973) appear, however, to have so far successfully obtained actual data on flare X-ray polarisation. The five bursts observed show polarisations between $20 \%$ and $40 \%$ (e.g. Figure 10 ) with some evidence of increasing polarisation with distance from the disc centre, (cf. Fig- 
ure 12) while the polarisation plane has been found to lie along the solar disc radius through the source.

Some problems of the theoretical interpretation of these data and their time evolution have been pointed out by Frost (1974a) and Korchak (1974). In particular the energy range observed is really too low since much of the radiation is thermal. Frost (1974a) has combined the $(0.6-1 \AA)$ polarisation data for the event of 1970 , November 5 at 0310 UT with OSO-5 hard X-rays (14-250 keV) and soft X-rays (2-8 $\AA$ ) as shown in Figure 10. Frost points out that polarisation peaks correspond well with

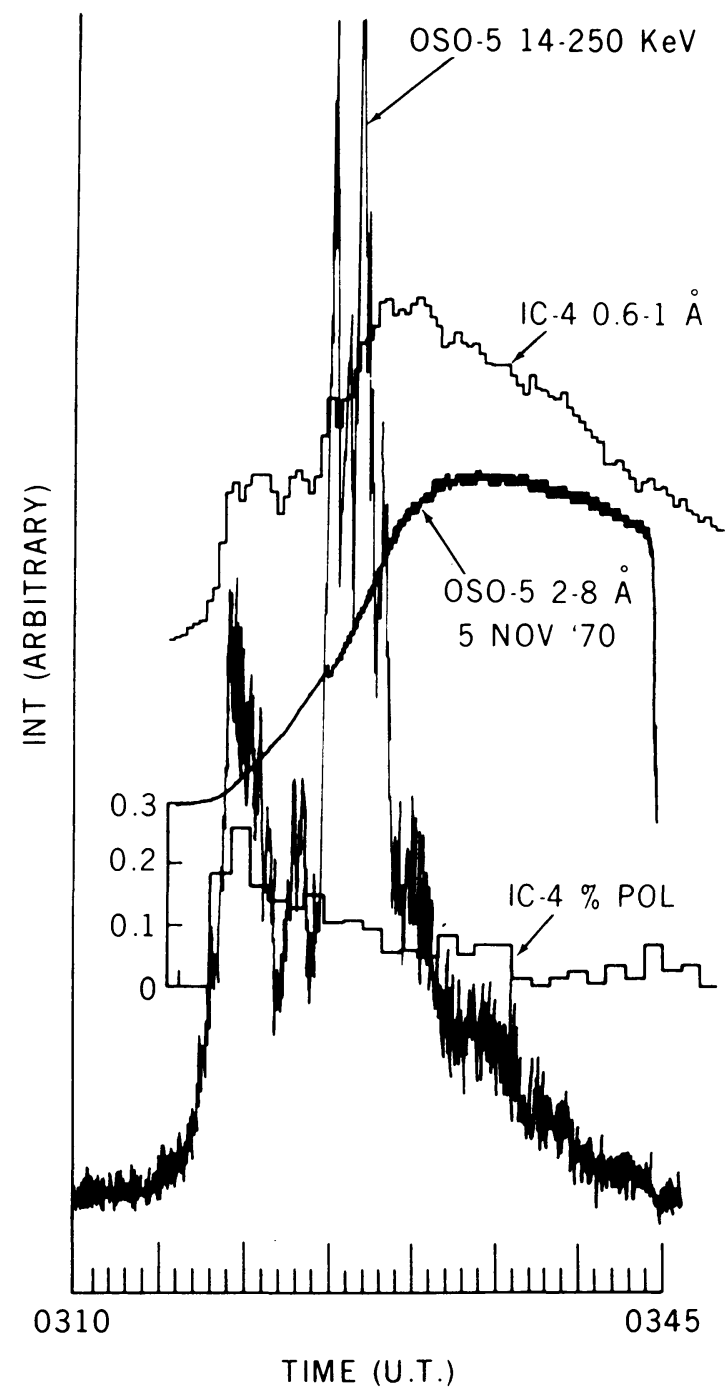

Fig. 10. Evolution of the polarisation in the flare of 1970, November 5 from Tindo et al. (1972b) compared to OSO-5 hard X-ray and soft X-ray data (prepared and provided by K. J. Frost, 1974b, see text for comments). 
hard X-ray intensity spikes early in the event but that the monotonic decay of the polarisation after 0315 UT may be due to the rising (unpolarised) thermal contribution and, therefore, that the $20 \%$ peak recorded must be regarded as a lower limit. Frost concludes that polarisation measurements must be made at higher energy and with high time resolution since both the degree and plane of polarisation may change between successive hard X-ray spikes (see also comments by Korchak (1974) on these problems).

In addition, Brown et al. (1974) have shown that some error may have been induced in the results of Tindo et al. due to their calibration procedure carried out by supposing the polarisation to be zero in the late (thermal) stages of the flare whereas even thermal flare X-rays may be somewhat polarized by photospheric backscattering. The extent of this error has not been evaluated satisfactorily (cf. Brown et al., 1974; Beigman and Vainstein, 1974) but future experiments would yield more easily interpretable results if they operated at higher energies and were laboratory calibrated.

Directional characteristics of bremsstrahlung X-ray sources are closely related to their polarisation and can be used for the same purpose - i.e. the inference of the source electron velocity distribution. Various contradictory attempts have been made to deduce the mean directivity of bursts by examining their distribution across the solar disc (e.g. Ohki, 1969; Pinter, 1969; Drake, 1971; Kane, 1974b; Datlowe, 1975). However, such burst distribution studies seem unlikely ever to lead to meaningful directivity data for, firstly, the maximum directivity expected from any model is less than a factor of 10, even at high photon energies (Elwert and Haug, 1971; Brown, $1.972 \mathrm{~b}$ ) while the dynamic range of intensities between different bursts exceeds a factor of $10^{3}$. Secondly, this is much beyond the dynamic range of any single detector so that strong selection effects are present in data samples. A proper statistical analysis of the problem requires use of the actual distribution of burst intensities but the spread in mean intensity for $N$ observed bursts will only decrease something like $1 / \sqrt{ } N$ so that a huge number of events must be observed before the spread in intrinsic intensities is averaged to much less than the directivity range sought (bearing in mind that the number of bursts is further diluted into a set of longitude intervals).

Distribution of spectral indices $\gamma$ across the disc has also been analysed - i.e. the directional characteristics of burst spectra. Kane (1974b) finds no significant variation across the disc while Datlowe (1975) reports increase in mean spectral index towards and over the limb for OSO-7 bursts. The interpretation of this result both in terms of its statistical reality and of its physical significance, if real, is unclear at present.

\section{(d) OTHER RELEVANT OBSERVATIONS}

Information on the spatial characteristics of hard X-ray flares is exceedingly sparse. Takakura et al. (1971) have obtained the only direct resolution of a hard X-ray flare, showing the emission to be localized (horizontally) near the core of the optical flare, but only with a resolution of about $1^{\prime}$ and in one dimension. In addition, there are a 
number of cases of occurrence of hard X-ray bursts from flares presumed behind the limb which set a lower limit to the altitude of at least some of the emission in those events $\left(\gtrsim 10^{4} \mathrm{~km}\right.$ - Datlowe, 1975). In one commonly quoted instance of a behindthe-limb burst, viz. 1969, March 30 (Kane, 1974a), however, this interpretation could be incorrect since this vast event extended over much of a solar hemisphere at radio frequencies (Wild and Smerd, 1972) and the X-ray source may have done likewise (Frost 1974b - private communication). A basic problem is that there is no way to distinguish purely coronal level emission from a small event and a small coronal component of a large event, when the flare itself is invisible.

Further limits are set by the interrelation of X-ray bursts to other flare flash-phase characteristics (cf. reviews by Kane, 1973; Brown, 1973b). In particular the very close synchronism of hard X-ray time profiles with (thermal) EUV bursts (Kane and Donnelly, 1971) which occur deep in the atmosphere $\left(n \gtrsim 10^{11} \mathrm{~cm}^{-3}\right)$ implies either that the X-ray source itself is at low altitude or that the two emissions are closely linked to a common energy supply, extended in altitude. Similar conclusions arise from associated optical flashes (Vorpahl, 1973, Zirin and Tanaka, 1973). On the other hand the correspondence of time structure of hard X-rays with soft X-rays flare development (Neupert, 1968) and with microwave bursts (e.g. Takakura, 1975) require a close coupling of the hard X-ray source with events high in the atmosphere also. A high altitude for the electron acceleration region itself seems to be well established by the lack of collisional distortion of interplanetary electron spectra down to a few $\mathrm{keV}$, as shown by Lin $(1974 a, b)$. The discrepancy $\left(\gtrsim \times 10^{3}\right)$ between the flux of electrons needed for the X-ray and synchronous microwave burst is well known (Takakura and Kai, 1966) and may be resolved by invoking the combined effects of microwave reabsorption, magnetic field distribution, and the high energy electron spectral cut-off (Takakura, 1973). Interplanetary electrons are also usually small in number (sometimes $\lesssim 0.5 \%$ ) compared to X-ray source electrons (e.g. Datlowe and Lin, 1973; Lin, $1974 \mathrm{a}, \mathrm{b})$, apparently due to magnetic confinement of the bulk of accelerated particles in the flare region. Nevertheless it seems an odd coincidence that both microwaves and interplanetary electrons yield similar numbers, both much smaller than that inferred from X-rays (cf. Section VI(c)).

\section{Inference of Mean Electron Spectra in Sources}

\section{(a) INTRODUCTION}

In general the inference of the spectrum of flare electrons at acceleration or the variation of their spectrum through the X-ray source is quite strongly model dependent cf. Section V. However, burst observations without spatial resolution do permit determination of a 'mean source electron spectrum' (Brown, 1971), independent of models, provided albedo and directional effects are neglected. Thus by defining

$$
F(E, t)=\frac{1}{\bar{n} V} \int_{V} n(\mathbf{r}, t) F(E, \mathbf{r}, t) \mathrm{d} V,
$$


where

$$
\bar{n}=\frac{1}{V} \int_{V} n(\mathbf{r}, t) \mathrm{d} V
$$

then Equation (1) becomes

$$
I(\varepsilon, t)=\frac{\bar{n} V}{4 \pi R^{2}} \int_{\varepsilon}^{\infty} F(E, t) Q(\varepsilon, E) \mathrm{d} E
$$

and $F(E, t)$ is the instantaneous space average of $F$ weighted with respect to the ambient plasma density $n$.

Equation (3) is then an integral equation to be solved for $F(E, t)$ with $I(\varepsilon, t)$ given and $Q(\varepsilon, E)$ as kernel. In practice $I(\varepsilon, t)$ is not known except after folding through an instrumental response and, even then, only in a discrete set of photon energy bands set by discriminator levels. A common procedure is to assume a functional form for $I(\varepsilon)$ (usually $A \varepsilon^{-\gamma}$ ) and to adjust the function parameters so as to optimize the $\chi^{2}$ fit of the calculated count rates to the data. This has the disadvantage that, although it optimises the fit for any chosen functional form, the functional form itself is somewhat arbitrary. (In the problem of distributed temperature fitting of soft X-ray spectra - e.g. Herring and Craig, 1973; Dere et al., 1974; Craig, 1974; Craig and Brown, 1974 - this problem is a crucial one since there exists no a priori basis for adoption of any particular fitting function.) A more satisfactory approach is first to deconvolute $I(\varepsilon)$ from the count rates, knowing the instrumental response. Problems of and optimum procedures for this step have been considered in detail by Hoyng and Stevens (1974) and applied to the hard X-ray burst problem by Hoyng et al. (1975), or at least a discrete representation of it. Then the remaining problem of solution of Equation (3) (or its matrix equivalent in the discrete case) is a matter of the basic physics of the bremsstrahlung process, independent of the instrument (except in so far as this partially determines the observational and discretisation errors).

\section{(b) THE INTEGRAL INVERSION PROBLEM}

From the theoretical viewpoint, one is interested in how $F(E)$ is related to different functional forms of $I(\varepsilon)$ - i.e. in the analytic inversion of Equation (3). Though such a solution may never be achievable from practical data in discrete form (i.e. without use of fitting functions), it is nevertheless of fundamental importance since it determines how accurately and unambiguously electron spectra can ever be determined from the spectra of their bremsstrahlung emission, however good the X-ray spectral resolution may be. That is, fundamentally, how good are bremsstrahlung X-rays as a measuring device for electron spectra?

The possibility of analytic solution of (3) depends of course on the form of the kernel $Q(\varepsilon, E)$. For the full cross-section expression with relativistic, Coulomb and other corrections included (Koch and Motz, 1959), no such solution is obtainable in simple form and the equation must be converted to an equivalent discrete set of linear 
equations and inverted numerically. The most complex kernel for which analytic solution seems possible (provided directional effects are neglected - i.e. assuming isotropy of source electron velocities), is the non-relativistic Bethe-Heitler ( $\mathbf{B H})$ formula which is fortunately also a good approximation below $100 \mathrm{keV}$ or so, namely (Koch and Motz, 1959)

$$
Q_{\mathrm{BH}}(\varepsilon, E)=\frac{K_{\mathrm{BH}} \log 1+\sqrt{1-\varepsilon / E}}{\varepsilon E} 1-\sqrt{1-\varepsilon / E},
$$

where $K_{\mathrm{BH}}=\zeta \times(8 \alpha / 3 \pi) r_{0}^{2} m c^{2}$, with $\alpha=$ fine structure constant, $c=$ velocity of light, $m$ and $r_{0}$ are the electron mass and radius, and $\zeta$ is the correction factor for the solar abundance of heavy ions $(\zeta \simeq 1.8$, Elwert and Haug, 1971).

The solution of (3), with (4) as kernel, has been shown by Brown (1971), via transformation to Abel's integral equation, to be

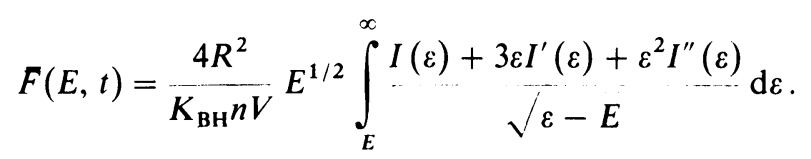

For the commonest case of a power-law, viz.

$$
I(\varepsilon)=A \varepsilon^{-\gamma}
$$

(with $A, \gamma$ functions of $t$ ), solution (5) reduces to

$$
F(E, t)=\frac{4 R^{2}(\gamma-1)^{2} B\left(\gamma-\frac{1}{2}, \frac{1}{2}\right)}{K_{\mathrm{BH}}} A E^{-\gamma+1},
$$

where $B$ is the beta function, or in numerical form * with $\varepsilon, E$ in $\mathrm{keV}$

$$
\begin{aligned}
\left.F(E, t) \text { (electrons } \mathrm{cm}^{-2} \mathrm{~s}^{-1} \mathrm{keV}^{-1}\right)= & \frac{6.7 \times 10^{50}}{\bar{n} V} \times \\
& \times E^{1 / 2} \int_{E}^{\infty} \frac{\left\{I+3 \varepsilon I^{\prime}+\varepsilon^{2} I^{\prime \prime}\right\}}{\sqrt{\varepsilon-E}} \mathrm{~d} \varepsilon
\end{aligned}
$$

in the general case and, for the power-law, in the same units

$$
F(E, t)=\frac{6.7 \times 10^{50}}{\bar{n} V}(\gamma-1)^{2} B\left(\gamma-\frac{1}{2}, \frac{1}{2}\right) A E^{-\gamma+1},
$$

where $I$ is in photons $\mathrm{cm}^{-2} \mathrm{~s}^{-1} \mathrm{keV}^{-1}$.

Other approximations have been used for $Q(\varepsilon, E)$ in the literature. In particular the approximation $Q \sim 1 / \varepsilon^{2}$ and that used by Kawabata et al. (1973) $(\sim 1 / \varepsilon E)$ are readily shown to give the same electron/X-ray spectral index relationship as (9), namely $\delta=\gamma-1$, for the power-law case, and to yield analytic inversions simpler than (5). However, both of these solutions involve a scale error in $F$ of factors up to three or

* Equations (8) and (9), and also (17), (19), (22), and (23), incorporate heavy ion correction $\zeta$ and correct a numerical error in Brown's (1971) paper. 
four, depending on $\gamma$, due to incorrect description of the behaviour of $Q$ in the region $\varepsilon \lesssim E \lesssim 2 \varepsilon$ which dominates the emission for the typically steep spectra involved. Though this factor is small in terms of the uncertainty of the total electron flux due to the unknown energy cut-off (cf. Sections III and VII), it is important in problems such as chromospheric heating by electrons where the low energy end of the spectrum is irrelevant (Brown, 1973a). Furthermore, in the case of spectra other than the powerlaw (6), these simpler approximations also yield the incorrect electron spectrum. For instance, Van Beek et al. (1974) have shown that a good fit to the X-ray spectral break, rather than a power-law with exponential cut-off, may be a pair of distinct power-laws joined at a sharply defined 'knee-energy'. Depending on just how 'sharp' this knee is, the contribution to solution (5) from the $I^{\prime \prime}$ term could dominate the others - a feature not present in the analytic solutions for simpler $Q(\varepsilon, E)$.

Thus, except for first approximations, it is wise to use the Bethe-Heitler result (5) for all analytic work.

\section{(c) THE MATRIX SOLUTION}

Present spectral resolution is not in fact capable of providing directly the data needed for use of the above solutions - e.g. of providing the second derivative of $I$ - without use of some smooth fitting functions. It is therefore important to consider the problem of solution of Equation (3) in discrete form - this procedure being also required when using more general (e.g. relativistic) cross-section formulae for which no analytic solution exists (cf. Elwert and Haug, 1970). That is $I(\varepsilon)$ is replaced by a set of $n$ discrete values $I_{i}=I\left(\varepsilon_{i}\right) i=1, n$ (in fact by $\int \hat{\Delta} \varepsilon_{i} I(\varepsilon) \mathrm{d} \varepsilon$, but Hoyng and Stevens, 1974, have shown how the optimum discrete set $I\left(\varepsilon_{i}\right)$ can be extracted from finite band data). Then $F$ is also replaced by a discrete representation $F_{j}\left(=F\left(E_{j}\right) \Delta E_{j}\right)$ where $j=1, m$ with $m \leqslant n$, inequality corresponding to a least squares fitting problem and equality to an exact problem, only the latter case being considered here. Thus

or

$$
\sum_{j=1}^{n} Q_{i j} F_{j}=I_{i}, i=1, n
$$

$$
[Q]\{F\}=\{I\},
$$

where $\{I\}\{F\}$ are the data and unknown $n \times 1$ vectors respectively and $[Q]$ is the (square) cross-section matrix, the formal solution being

$$
\{F\}=\left[Q^{-1}\right]\{I\} \text {. }
$$

The accuracy of matrix solution (11) is limited by the usual numerical instabilities in solution of linear equations (e.g. Fox and Mayers, 1968; Householder, 1964) i.e. by magnification of small errors in $\{I\}$ by large entries in $\left\{Q^{-1}\right]$ resulting in larger relative errors in $\{F\}$. In some cases these are so large as to render the solution obtained meaningless, the severity of the problem depending both on the form of the matrix $[Q]$ and of the data $\{I\}$. Considering first the effects of the matrix itself a convenient measure of the likelihood of such inherent instability is the matrix condi- 
tion number $N$ (e.g. Householder, 1964) given by $N=\|Q\| \times\left\|Q^{-1}\right\|$. $\|A\|$ is any convenient norm of a matrix $A$ such as $\left[\sum_{i, j} a_{i j}^{2}\right]^{1 / 2} / n^{1 / 2}$ which implies $N=1$ for the numerically ideal case of the unity matrix (or any scalar multiple of it). The actual case for the bremsstrahlung cross-section matrix $Q$ is an upper triangular matrix with small diagonal elements - far from diagonal and so potentially ill-conditioned. As a simple illustration, if data on $I$ are obtained at $\varepsilon_{i}=20,40$ and $80 \mathrm{keV}$ and represented in terms of electron fluxes at $E_{j}=25,50$ and $100 \mathrm{keV}$, then with convenient scaling, the Bethe-Heitler cross section gives

$Q=\left[\begin{array}{ccc}19.2 & 20.6 & 14.4 \\ 0 & 4.82 & 5.08 \\ 0 & 0 & 1.21\end{array}\right]$ and so $Q^{-1}=\left[\begin{array}{llr}0.052 & -0.224 & 0.310 \\ 0 & 0.207 & -0.870 \\ 0 & 0 & 0.828\end{array}\right]$

which implies $N \simeq 21.6$ (as against $N=1$ for a unity $3 \times 3$ ) corresponding to a large inherent instability in the set of Equations (19) - i.e. in the derivation of electron from photon spectra. No improvement of spectral resolution (within a fixed $\varepsilon$ range) can improve this situation since the introduction of more and more rows into $Q$ merely increases their linear dependence and hence the ill-conditioning. The conclusion is that, for general data $\{I\}$, high accuracy is needed for solution $\{F\}$ to be guaranteed as meaningful, the necessary accuracy increasing with the dimension of $\{I\}$.

The actual severity of the consequences of an ill-conditioned matrix depends, however, on the problem itself - i.e. on the data (thus for example the errors introduced in $F_{j}$ from $I_{i}, i \neq j$, by large off-diagonal elements of $Q^{-1}$ may in fact be relatively small if $I_{i}$ is itself small compared to $I_{j}$ ). In particular if, in (10), the $F_{j}$ decrease rapidly with increasing $j$ (i.e. $E_{j}$ ) then the effect is essentially to damp the magnification of errors inherent in the matrix form itself. This condition is probably satisfied by the powerlaw and other steeply decreasing functions so that if one has grounds for expecting such a spectrum (other than the $\mathrm{X}$-ray data itself) then the instability problem need not to be so serious in practice. What is not true, however, is the usual supposition that, because substitution of (e.g.) a power-law for $\{F\}$ in (10) yields a 'good' fit to $\{I\}$ the power-law is necessarily near the true electron spectrum. It may merely show that the calculated X-ray spectrum is rather insensitive to the source electron spectrum as confirmed by the sensitivity of the inverse analytic expression (5), to $I(\varepsilon)$. One is therefore forced to the conclusion that, in general, bremsstrahlung X-rays are not a very accurate meter for electron spectra unless one has a pretty good idea beforehand what form these electron spectra take. Confidence in the ubiquitous use of power-laws for this purpose, other than as an act of faith in a model, then rests largely on consistency of this model with other related flare data. For this reason, in evaluating flare particle acceleration models, the constraint of reproducing a power-law spectrum should perhaps not be taken overseriously.

\section{(d) THE PROBLEM OF THE THERMAL X-RAY SPECTRUM}

It is apt to mention the analogous problem of inference of the temperature distribution in the hot flare plasma from soft X-ray spectral measurements. This is important 
for correct assessment of the total energy in the soft X-ray flare plasma (Craig 1974) and for its influence on the hard X-ray energy range (Brown 1974 and Section VI of this review). In this problem there is no definitive guide whatsoever, from theory or observation, as to what functional form the differential emission measure may take. Consequently various entirely arbitrary fitting functions have been used (e.g. Dere et al., 1974) with parameters adjusted to 'optimize' the fit to observations. However, the equations involved are exceedingly ill-conditioned (Craig and Brown, 1974) and it is far from clear whether the fitting functions used are physically meaningful or merely suitable damping functions for the inherent instabilities of the problem. These difficulties arise by the nature of the thermal continuum emission function compounded by the substantial contribution from X-ray lines, and are as important for cosmic as for solar X-ray studies. The most soundly based attack on the problem and its consequences is that initiated by Herring and Craig (1973) and continued by Craig (1974 and currently being pursued further by Craig and Brown (1974).

\section{(e) X-RAY SPECTRA FROM A POWER-LAW ELECTRON SPECTRUM}

Conversely to the conclusions of IV(c), if one does favour the power-law as a likely form for the electron spectrum, it is important to know whether the X-ray observations are in fact consistent with it, even if not precise enough to establish it. That is, whether any observed deviations from an X-ray power-law - such as the high energy break can be explained in a natural way other than in terms of deviation of the electron acceleration spectrum itself from a power-law. Though a number of factors relating to this question are discussed further in Sections 5 and 6, the chief causes of such deviations may be summarized here.

\section{(i) The Albedo Contribution}

Tomblin (1972) and Santangelo et al. (1973) have investigated the spectrum of photospheric albedo photons and their influence on the spectrum of total burst X-rays. At low energies $(\lesssim 15 \mathrm{keV})$ the albedo contribution drops off due to photoelectric absorption. At high photon energies the albedo contribution is reduced by the relativistic anisotropy of Compton scattered photons and by the increasing energy loss of photons in the Compton scattering process. The net effect is a downward concavity (steepening) of the total $\mathrm{X}$-ray spectrum around the $40 \mathrm{keV}$ range.

\section{(ii) Directivity Effects}

Since the bremsstrahlung cross-section is anisotropic (Koch and Motz, 1959), if the source electron velocity distribution is not isotropic, the $\mathrm{X}$-ray emission in general varies both in intensity and spectrum with direction (Elwert and Haug, 1970). These effects are particularly important at high photon energies due to relativistic beaming (Elwert and Haug, 1971). Petrosian (1973) has calculated that, for the case of a collimated electron stream moving vertically toward the photosphere, the emission spectrum should show a break above about $100 \mathrm{keV}$, and proposed that this cross-section effect may explain the observation of a high energy cut-off. This result has not been noted 
by previous authors on this topic (Elwert and Haug, 1971; Brown, 1972b) and it is not clear to what extent it is dependent on the different cross-sections used. Furthermore, Brown (1972b) has previously considered exactly the same problem but included collisional scattering of the electron stream (neglected by Petrosian) which reduces all anisotropic effects, and found no spectral break. Since the actual geometry of the source is quite unknown, however, the possibility of a spectral correction for directional effects should be borne in mind.

\section{(iii) Model Dependent Effects}

Examination of definition (2) shows that if the source plasma density $n$ is homogeneous $(n \neq n(\mathbf{r}))$ or if the energetic electron spectrum is homogeneous $(F \neq F(\mathbf{r}))$ then the mean effective source spectrum (2) can be identified with the true spectral distribution of all electrons in the source. If, however, both $n$ and $F$ are inhomogeneous then in general the mean source spectrum (2) differs from the true spectrum in the sense that if electrons in some energy range move in part of the source where $n$ is higher than elsewhere, then that part of the X-ray spectrum is enhanced (e.g. Brown, 1972a). Since no spatially resolved data are available, the form of such differences is dependent on the model adopted (see below).

Secondly, in models where the energy loss time of electrons is small, it is necessary to distinguish the spectrum of continuously accelerated electrons (before injection) and the instantaneous spectrum of electrons in the source due to the effects of the energy loss on the latter, as considered in $\mathrm{V}(\mathrm{a})$. This may cause deviations from a power-law (e.g. Brown, 1973d) as well as changing the power-law index (Brown, 1971).

In consequence of these three effects, it must be concluded that though there are doubts about the accuracy with which one can truly infer electron spectra, there is no conclusive observational evidence inconsistent with the power-law as a model of the entire electron spectrum at acceleration.

\section{Burst Interpretation and Source Models}

Discussions of the three main existing source models, viz. thick- and thin-target and electron trap, have been presented recently by Hudson (1973), Lin (1974), Brown (1973b), Takakura (1973) and Kane (1974a) while the possible importance of thermal emission in the hard X-ray range has been discussed by Brown (1974) and by Kahler (1975) in these Proceedings (cf. also VI(b) below). Here I will try to bring this controversy over models up to date, present the outstanding problems of these models, and speculate on how these might be resolved.

\section{(a) THE THICK TARGET MODEL}

This model postulates the injection of energetic electrons from a coronal source into the dense chromosphere where the bulk of bremsstrahlung X-rays are then generated (De Jager and Kundu, 1963; Arnoldy et al., 1968: Acton, 1968; Brown, 1971, 1972b: 
Hudson, 1972; Syrovatskii and Shmeleva, 1972; Petrosian, 1973; Brown and McClymont, 1974). The electrons are of course totally collisionally absorbed on very short time scales (Schatzman, 1966; Brown, 1973a) so that the bremsstrahlung target is thick and, furthermore, the time profile of the resulting burst is governed entirely by the injection rate of electrons from their source.

Thus, with reference to Equation (1), time variations in $I(\varepsilon, t)$ are entirely attributed to variations in $F(E, \mathbf{r}, t)$ due to electron source modulations, the minimum time scale of burst variation being set by the collisional loss term in $\mathrm{d} F / \mathrm{d} t$, electron escape being non-existent in this model (cf. Lin, 1974a). Since in fact the collisional losses, and hence the bremsstrahlung emission, occur almost entirely over about one chromospheric scale height (Brown, 1972b, 1973a; Brown and McClymont, 1974), this lower limit is in the millisecond range - well below available time resolution.

Satisfactory explanation of burst time profiles in the thick-target model is thus entirely a problem for the electron acceleration mechanism itself. This area of research, perhaps more than any other, is currently in need of intensive theoretical and laboratory investigation both in terms of the capabilities of any acceleration mechanism to produce the complex fine structure in bursts, and of quantitative analysis of burst time profiles to yield any basic characteristics of the electron flux and spectrum at acceleration (cf. Kane and Anderson, 1970; Hoyng et al., 1975) which could give any clue to the acceleration mechanism (cf. Vorpahl and Takakura, 1974). For the present it is generally merely assumed that the mechanism is capable of producing observed burst profiles.

\section{(i) Polarization and Directivity}

Following on the first descriptions of the possibility and importance of flare bremsstrahlung polarization (Korchak, 1967a, b, Elwert, 1968) Elwert and Haug (1970, 1971) and Haug (1972) have developed in detail the methodology for calculation of both the polarisation and directivity of bremsstrahlung from electron streams spiralling in a uniform magnetised plasma, emphasising the need to use fully relativistic cross-sections, though the electrons themselves are only semi-relativistic. Application of these methods to realistic source models are very laborious, involving, in general, four dimensional integration - along a curved guiding field with variable plasma density, as well as over the electron azimuth, pitch-angle, and energy distributions. Nevertheless, as recently emphasised by Korchak (1974), this is the only way in which satisfactory predictions are obtainable. The only published results for a specific model are those of Brown (1972b) for the case of a thick-target model with a purely vertical guiding field and an electron stream injected vertically but with collisional modification of the electron energies and pitch angles in the target. The plane of maximum intensity is that containing the source and the solar disc centre while the degree of polarisation increases from zero at disc centre to about $30 \%$ near the limb, with a slight energy dependence, as shown in Figure 11 (together with results neglecting scattering). These predictions are in agreement with the tentative observational results 
of Tindo et al. (1970, 1972a, b, 1973) both in terms of the polarisation plane and the dependence on solar central distance (Figure 12).

The directivity of this thick-target model is shown in Figure 13. When scattering of the electron beam is included, the directivity is seen to be quite small $(\simeq 3)$ except at high photon energies but in all cases is in the sense of an increasing burst intensity toward the limb - a trend predicted by earlier qualitative descriptions (Ohki, 1969; Pinter, 1969). As discussed in III(c), however, no satisfactory directivity data are

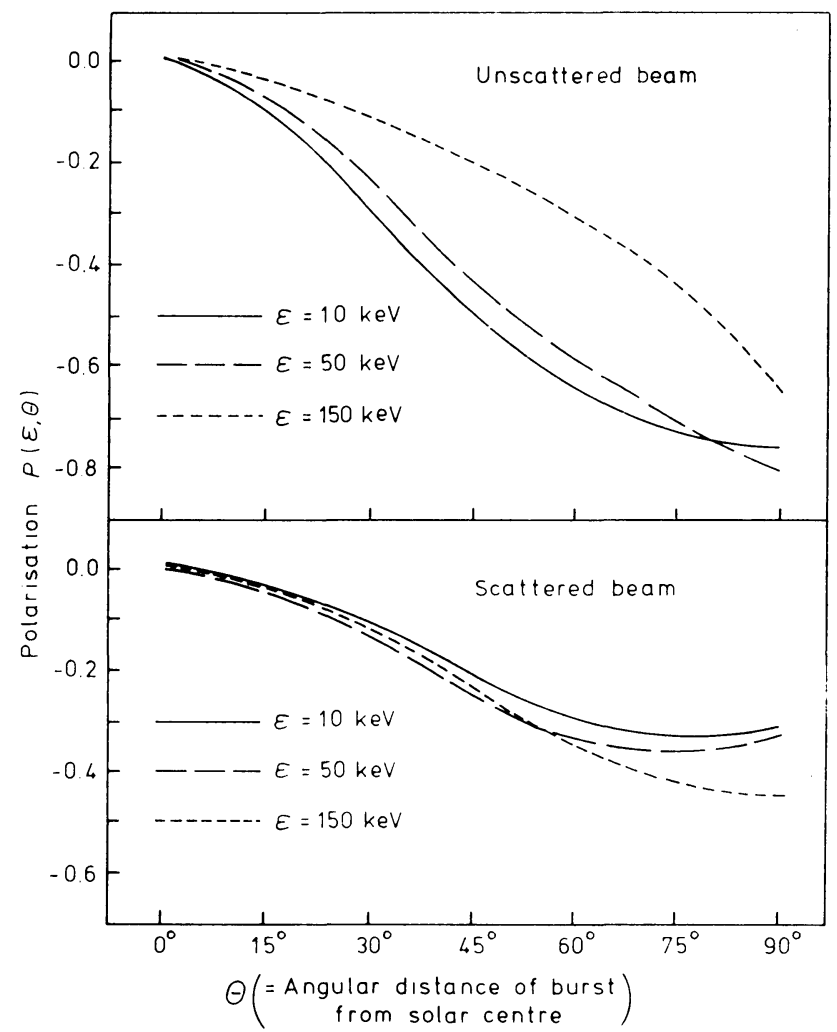

Fig. 11. Polarisation of X-rays, for the thick-target source geometry analysed by Brown (1972b) (with and without collisional scattering of the electron beam) as a function of flare location and energy of observation.

available for comparison purposes and it is unlikely that the model can be tested on this basis until 'stereo' observations are made of individual bursts.

Brown's (1972b) analysis (cf. Petrosian, 1973) predicts a burst spectral index which decreases from center to limb, in conflict with the observational claims of Datlowe (1975). An important piece of analysis will be to consider the modification of the emission directivity by the photospheric albedo for this and other models with an anisotropic primary source. The effect will be greatest for the thick target model in which the bulk of X-rays is emitted downwards. 


\section{(ii) Spatial Distribution of the Emission}

Brown and McClymont (1974) have recently made quantitative predictions of the height distribution of the thick-target emission and find, as expected, that it virtually all emanates from the narrow range of column depths ( $\simeq$ one scale height) over which the electrons dump most of their energy collisionally. Association of this distribution with geometric height depends, however, on adoption of some model of the flaring atmosphere. When a quiet atmosphere model is used, the emission is highly localised

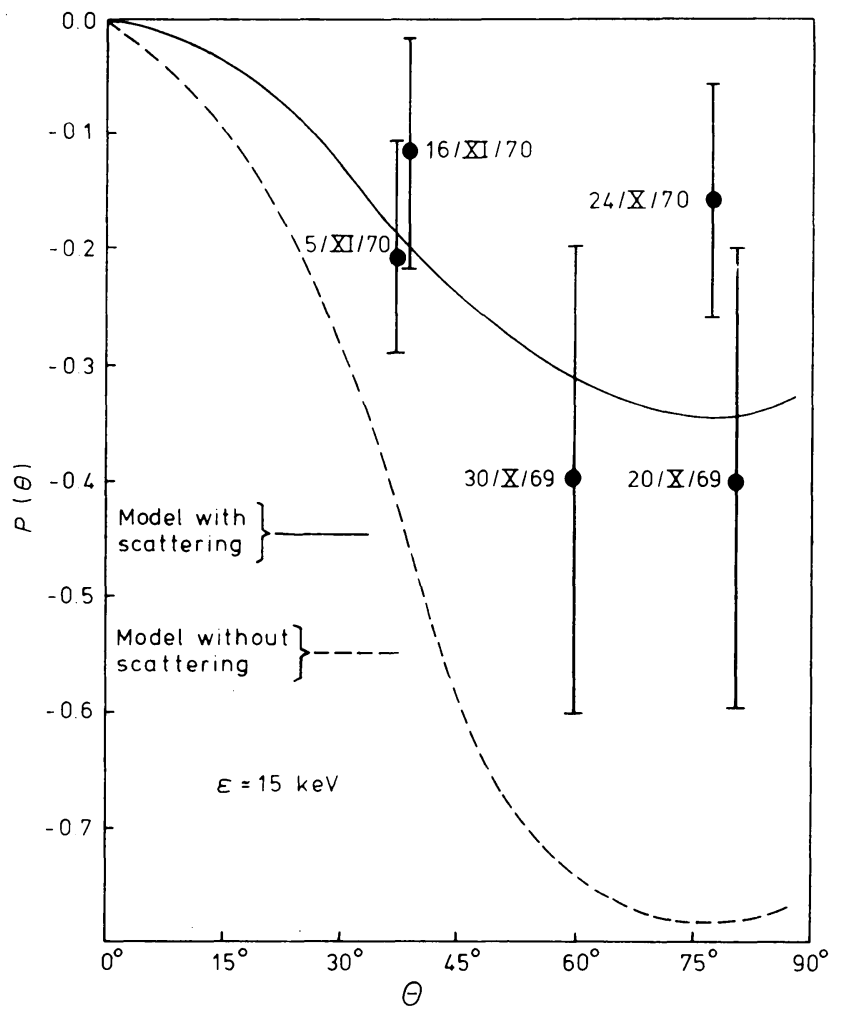

Fig. 12. Comparison of the predictions of thick-target polarisation at $15 \mathrm{keV}$ from Brown (1972b) with the observations of five bursts obtained by Tindo et al. $(1970,1972 \mathrm{a}, \mathrm{b})$.

at $\$ 1000 \mathrm{~km}$ above the photosphere, in satisfactory agreement with the excellent correlation found between hard X-ray time profiles and those of the EUV and optical flares, known to be deep in the atmosphere. Though this correlation might only indicate a common energy source of the different emissions, rather than their spatial identity, it is doubtful whether any energy source, other than the fast electron streams themselves, is capable of producing the observed synchronism (Brown, 1973b). On the other hand it has been argued by Kane (1974a) and others that, if behind-the-limb events really do indicate a source altitude $\geqslant 10^{4} \mathrm{~km}$, then they are incompatible with 
a thick-target description. Brown and McClymont (1974) have, however, pointed out that in some, and especially in large, flares the distribution of plasma in the atmosphere is radically altered by the flare itself and specifically that the $10^{16} \mathrm{gm}$ of material ejected from an area of $10^{19} \mathrm{~cm}^{2}$ at more than $10^{3} \mathrm{~km} \mathrm{~s}^{-1}$ in the flash of a large flare (Sweet, 1969) is a thick plasma target to electrons in the X-ray energy range up to at least $70 \mathrm{keV}$. Hence the mass motion in an explosive flare is quite sufficient to raise

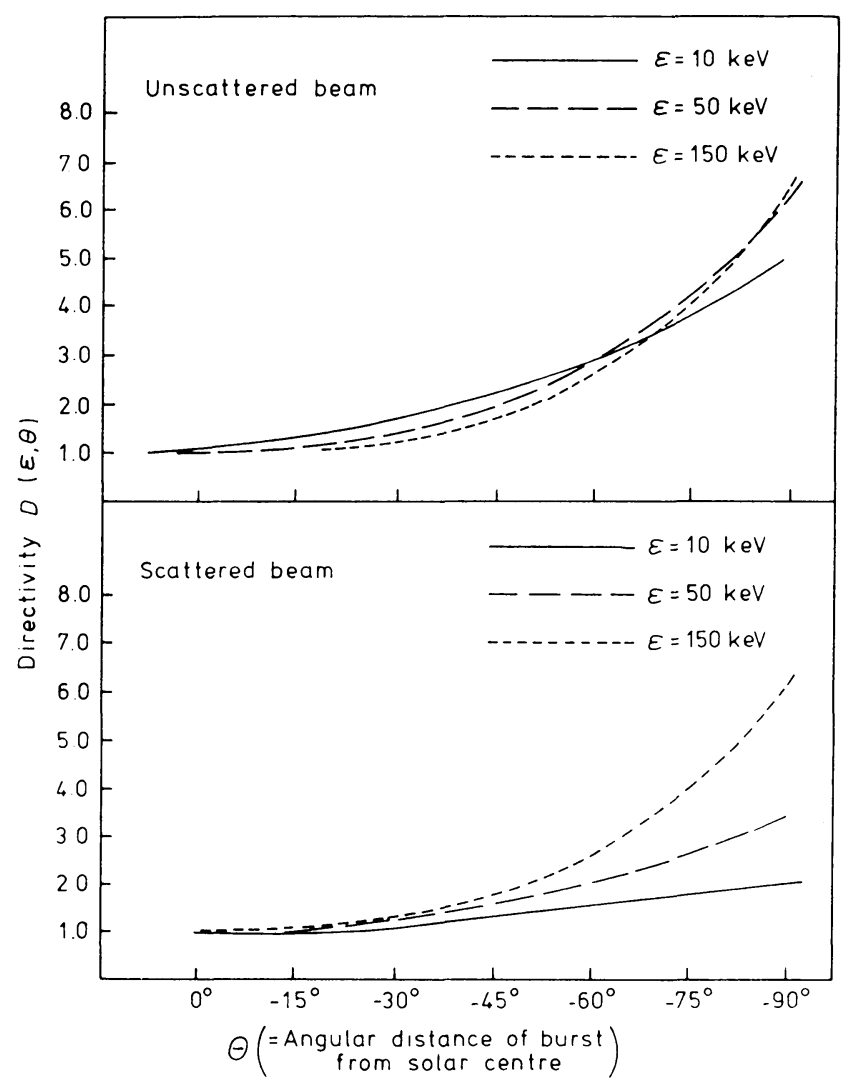

Fig. 13. Directivity of X-ray emission from Brown's (1972b) thick-target model, as a function of photon energy and flare location, defined relative to a flare at disc centre.

the thick target plasma to visibility above the limb within time scales (10-100 s) shorter than the duraction of a large burst. Satisfactory tests in this direction may however, only be possible with the advent of a hard X-ray heliograph.

(iii) The Electron Acceleration Spectrum

An important feature of the thick-target model is the distinction between the electron injection spectrum and the mean electron spectrum in the source region (Brown, 1971 and subsequent authors). Since the electron lifetime is very short compared to the 
observational resolution, it proves possible to express (1) in the form

$$
I(\varepsilon, t)=\frac{1}{4 \pi R^{2}} \int_{E_{0}=\varepsilon}^{\infty} \mathfrak{F}\left(E_{0}, t\right) \int_{E=\varepsilon}^{E_{0}} \frac{n Q v \mathrm{~d} E}{(\mathrm{~d} E / \mathrm{d} t)_{\mathrm{TOT}}} \mathrm{d} E_{0},
$$

where $\mathfrak{F}\left(E_{0}, t\right)$ is the acceleration spectrum - i.e. the number of electrons injected per s per unit $E_{0},(\mathrm{~d} E / \mathrm{d} t)_{\text {Tот }}$ is the total energy loss rate of an electron of injection energy $E_{0}$ when it has decayed to energy $E$ and $n$ is the local plasma density at that point. Due to the high density $n$ involved, there is no doubt that collisional energy losses entirely dominate the synchrotron and inverse Compton contributions to $(\mathrm{d} E / \mathrm{d} t)_{\text {To }}$. It is further generally assumed (Brown, 1971; Kane, 1973; Lin, 1974a, b) that collective losses to plasma wave generation can also be neglected on the grounds that the electron beam is dilute. If this is valid, then

$$
(\mathrm{d} E / \mathrm{d} t)_{\text {TOт }} \simeq{ }_{E}^{K} n v,
$$

where $K=2 \pi e^{4} \Lambda$ and $\Lambda$ is the effective Coulomb logarithm, so that (13) reduces to

$$
I(\varepsilon, t)=\frac{1}{4 \pi R^{2}} \int_{\varepsilon}^{x} \mathfrak{F}\left(E_{0}, t\right) \int_{\varepsilon}^{E_{0}} \frac{E Q(\varepsilon, E)}{K} \mathrm{~d} E \mathrm{~d} E_{0}
$$

which is independent of the source geometry provided $K$ is a function of $E$ only, and in fact a slowly varying one (Brown, 1973d has considered the effect of decreasing hydrogen ionisation with increasing depth in a thick flare target and finds that, due to $K$ decreasing with ionisation, the high energy end of the X-ray spectrum is somewhat enhanced). Again using the Bethe-Heitler formula for $Q(\varepsilon, E)$ it proves possible (Brown 1971) to solve (15) analytically for $\mathfrak{F}\left(E_{0}, t\right)$ to give

$$
\mathfrak{F}\left(E_{0}\right)=-\frac{4 K R^{2}}{K_{\mathrm{BH}}} \frac{1}{E_{0}^{3 / 2}} \int_{E_{0}}^{\infty} \frac{\varepsilon}{\sqrt{\varepsilon-E_{0}}}\left\{4 I^{\prime}+5 \varepsilon I^{\prime \prime}+\varepsilon^{2} I^{\prime \prime \prime}\right\} \mathrm{d} \varepsilon
$$

or

$$
\begin{aligned}
& \mathfrak{F}\left(E_{0}\right)\left(\text { electrons s }{ }^{-1} \mathrm{keV}^{-1}\right)=-2.0 \times 10^{33} E_{0}^{-3 / 2}(\mathrm{keV}) \int_{E_{0}}^{\infty} \frac{\varepsilon}{\sqrt{\varepsilon-E_{0}}} \times \\
& \times\left\{4 I^{\prime}+5 \varepsilon I^{\prime \prime}+\varepsilon^{2} I^{\prime \prime \prime}\right\} \mathrm{d} \varepsilon
\end{aligned}
$$

which, in the particular case of power law (6) reduces to

$$
\mathfrak{F}\left(E_{0}\right)=\frac{4 K R^{2}}{K_{\mathrm{BH}}}(\gamma-1)^{2} B\left(\gamma-\frac{1}{2}, \frac{1}{2}\right) A E_{0}^{-\gamma-1}
$$

or, in numerical form,

$$
\begin{aligned}
\left.\mathfrak{F}\left(E_{0}\right) \text { (electrons s}{ }^{-1} \mathrm{keV}^{-1}\right)=2.0 \times 10^{33} & (\gamma-1)^{2} \times \\
& \times B\left(\gamma-\frac{1}{2}, \frac{1}{2}\right) A E_{0}^{-\gamma-1},
\end{aligned}
$$


where $E_{0}$ is in $\mathrm{keV}$ and $A$ such that $A \varepsilon^{-\gamma}$ is in $\mathrm{cm}^{-2} \mathrm{~s}^{-1} \mathrm{keV}^{-1}$ with $\varepsilon$ in $\mathrm{keV}$. Equation (19) shows that the thick-target electron injection spectrum is two powers steeper than the mean spectrum (7), a result with important consequences for the energy and number of electrons contained in the extrapolated low energy end of the electron spectrum. In addition, Datlowe and Lin (1973) have shown how it might be used as a test of the model, if the interplanetary electron spectrum can be taken as that of the electrons at acceleration. Their results for one small flare show an interplanetary electron index $\delta$ nearer to $\gamma-1$ (as predicted by the thin-target model - cf. Section $V(b)$ ) than to $\gamma+1$ (as predicted above for the thick-target). On the other hand, as pointed out by McClymont and Brown (1974), the fact that the interplanetary electrons in that event comprise $\$ 0.4 \%$ of the number of electrons in the X-ray flare (Datlowe and Lin, 1973) puts the identification of their spectrum with that of the bulk of accelerated electrons in considerable doubt, since the few escaping particles, are, a priori, exceptional. Thus one cannot be sure at present whether energy dependent escape probability, or modification by plasma wave losses of the spectrum of electrons passing through the corona (cf. Section $V(b)$ ) may explain this discrepancy.

One major gap in our understanding of the thick-target and other electron stream models is whether and how the streams can in fact penetrate the plasma target against two stream instability losses to plasma waves. Though, as noted by Brown (1971), Lin (1974a, b) and Kane (1974a), the growth rate of such energy losses decreases as the stream becomes more dilute compared to the plasma, the growth time may still be small compared to the burst duration. Even without analysis of that problem, however, it is easy to see how radically the thick target model would be changed if collective losses were dominant. Firstly, any increase in $(\mathrm{d} E / \mathrm{d} t)_{\text {тот }}$ in (13) would proportionally reduce the efficiency of the thick-target as an X-ray emitter (since electron lifetimes would be reduced), thus worsening the already considerable problem of electron number and energy requirements (Section VI). Secondly, the energy dependence of collective losses certainly differs from the collisional form (14) so that the relationship between the X-ray and the electron acceleration spectra (17) will no longer hold. Thirdly, the spatial distribution of the emission would be shifted higher in the atmosphere, and finally, since decollimation of streams is an even more important effect of the two stream instability, both the polarisation and directivity would be greatly reduced.

\section{(b) THE THIN-TARGET MODEL}

Motivated by the observations of behind-the-limb bursts and of the X-ray/interplanetary electron spectral index relationship, Datlowe and Lin (1973) have proposed a model in which the hard X-ray burst time profile is again produced by continuous modulation of an electron source but in which the electrons stream upward through a (thin-target) coronal region. Since, however, as argued in Section V(a) these observations may actually be compatible with the thick-target, it is necessary to examine the thin-target predictions of other observable quantities and to weigh the two models in terms of their compatibility with overall flare requirements.

As regards reproducing observed time profiles, the same remarks apply to the thin- 
target as already made for the thick-target except that the lower limit to fine time structure is set in the thin-target not by the energy loss time (which $\rightarrow \infty$ ) but rather by the escape (or transit) time of electrons through the thin-target layer. This time may not be as small as in the thick-target since the target thickness cannot be less than the atmospheric scale height ( $\simeq 10^{10} \mathrm{~cm}$ in the corona) but is certainly small enough to be compatible with present observations.

\section{(i) Polarisation and Directivity}

No-one has specifically considered these aspects of the thin-target model but results will clearly depend on the collimation of the upward moving electron stream and so on the form of the guiding field, of the initial acceleration and of collective scattering processes. Upper limits may, however, be set from the results for a purely radial stream (cf. Elwert and Haug, 1971; and Haug, 1972). By symmetry, the polarisation would evidently be zero at the disc centre and increase to the limb as shown in Figure 14, the plane of

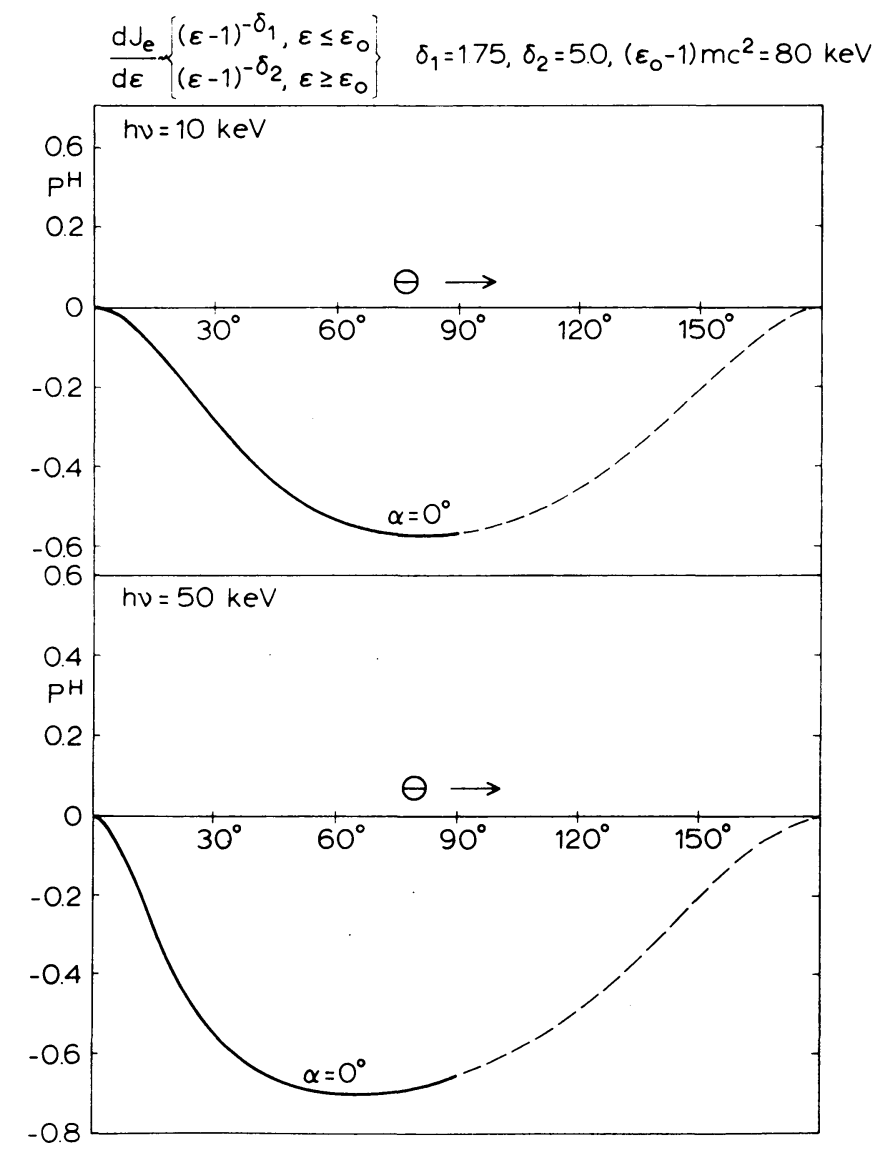

Fig. 14. Upper limit to the polarisation expected from the thin target model of Datlowe and Lin (1973) at 10 and $50 \mathrm{keV}$ as a function of flare location, based on Haug (1972). $\theta$ is the distance from the solar centre, so dotted portions are for flares behind the limb. 
polarisation being along the solar radius through the source. Figure 15 shows the maximum directivity of the model at $50 \mathrm{keV}$ with a maximum at $30-60^{\circ}$ from the disc centre then slight to severe darkening toward the limb depending on electron ' $k$ nee' energy $E_{0}$ (due to relativistic forward beaming of photons).

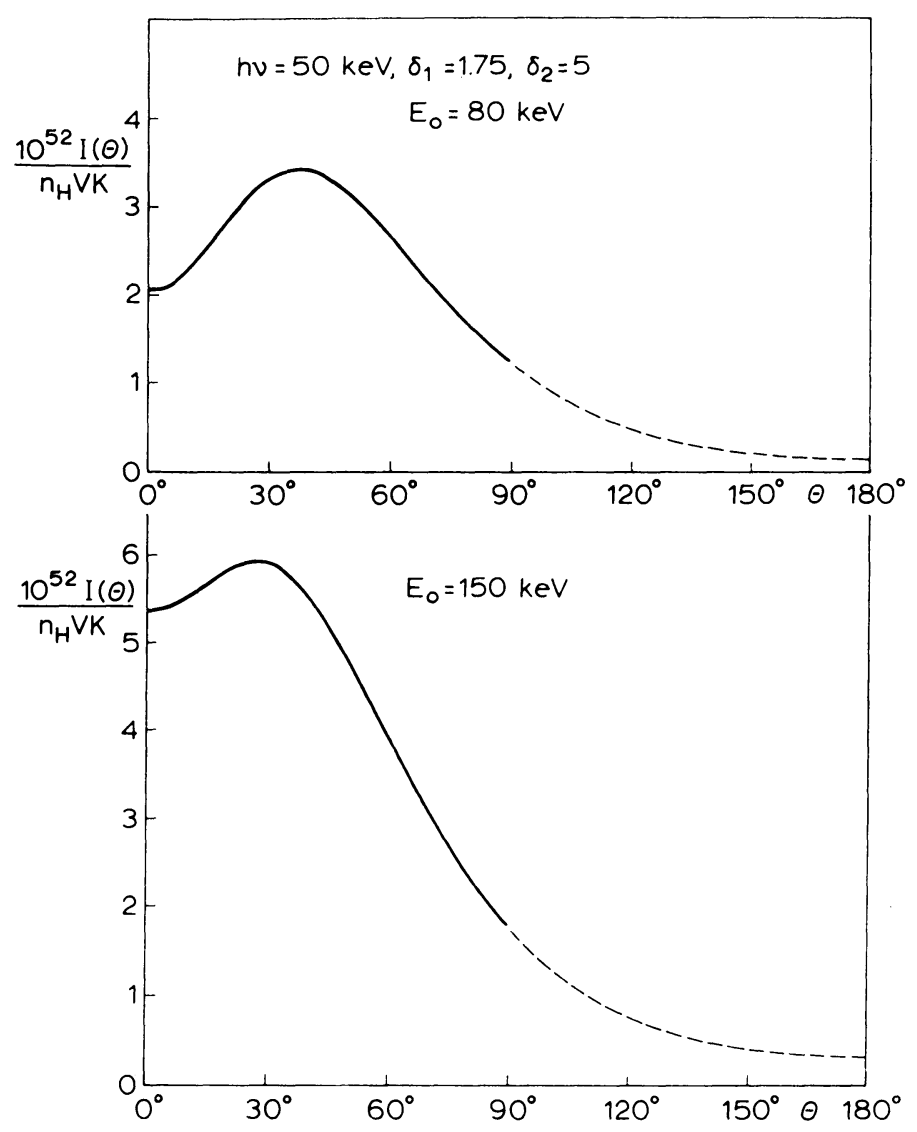

Fig. 15. Upper limit to relative variation of total burst intensities at $50 \mathrm{keV}$ across the disc in the thin-target model, (based on Elwert and Haug, 1971). $E_{0}$ is the electron spectrum 'knee-energy' (cf. Figure 14).

(ii) Electron Acceleration Spectrum

Since electrons passing through a thin-target do not undergo significant energy losses, the electron spectrum in the emitting layer is identical to the acceleration spectrum. Thus, in terms of a one-dimensional model, source Equation (1) simplifies (since $F \neq F(\mathbf{r}))$ to

$$
I(\varepsilon, t)=\begin{gathered}
\Delta N \\
4 \pi R^{2}
\end{gathered} \int_{\varepsilon}^{\infty} \mathfrak{F}\left(E_{0}, t\right) Q(\varepsilon, E) \mathrm{d} E_{0},
$$


where $\mathfrak{F}\left(E_{0}, t\right)$ is the total number of electrons per unit $E_{0}$ accelerated (and passing through the entire target area) per s and $\Delta N$ is the target 'thickness' in terms of ambient protons per $\mathrm{cm}^{2}$ column. Again in the Bethe-Heitler approximation the solution is

$$
\mathfrak{F}\left(E_{0}, t\right)=\frac{4 R^{2}}{K_{\mathrm{BH}} \Delta N} E_{0}^{1 / 2} \int_{E_{0}}^{\infty} \frac{\left\{I+3 \varepsilon I^{\prime}+\varepsilon^{2} I^{\prime \prime}\right\}}{\sqrt{\varepsilon-E_{0}}} \mathrm{~d} \varepsilon
$$

or

$$
\mathfrak{F}\left(E_{0}, t\right)\left(\mathrm{s}^{-1} \mathrm{keV}^{-1}\right)=\frac{6.7 \times 10^{50}}{\Delta N\left(\mathrm{~cm}^{-2}\right)} E_{0}^{1 / 2}(\mathrm{keV}) \int_{E_{0}}^{x} \frac{\left\{I+3 \varepsilon I^{\prime}+\varepsilon^{2} I^{\prime \prime}\right\}}{\sqrt{\varepsilon-E_{0}}} \mathrm{~d} \varepsilon
$$

and for power law (6)

$$
\mathfrak{F}\left(E_{0}, t\right)=\frac{6.7 \times 10^{50}}{\Delta N\left(\mathrm{~cm}^{-\overline{2}}\right)}(\gamma-1)^{2} B\left(\gamma-\frac{1}{2}, \frac{1}{2}\right) A E_{0}^{-\gamma+1}
$$

with $\varepsilon, E_{0}$ in $\mathrm{keV}$ throughout and $I$ in $\mathrm{cm}^{-2} \mathrm{~s}^{-1} \mathrm{keV}^{-1}$.

The limit to $\Delta N$ for which a target becomes classed as 'thin' is to some extent arbitrary of course but $\$ 30 \%$ 'collisionally thick' at $5 \mathrm{keV}$ and above must be an upper limit, implying $\Delta N \lesssim 10^{19} \mathrm{~cm}^{-2}$. Due to the low plasma density, however, collective energy losses are very much harder to circumvent in a thin than in a thick target. E.g. the large event of 1972, August 4 (Hoyng et al., 1974) requires $\simeq 10^{37}$ electrons s ${ }^{-1}$ even above $25 \mathrm{keV}$ and so a stream density (over $10^{19} \mathrm{~cm}^{2}$ ) $\gtrsim 10^{8} \mathrm{~cm}^{-3}$, which is not small compared to that of the coronal plasma. Unless a means can be found of stabilising the resulting two stream instability (as required for type III radio bursts - e.g. Smith, 1974), the coronal thin-target plasma would have to be allocated a very small $\Delta N$ indeed to remain 'thin' for total energy losses and the thin-target efficiency problem (see below) would be made much worse. Even if such stabilisation can be established, it is again unclear, as for the thick-target, how the stabilisation process may modify the electron spectrum after acceleration and hence vitiate the assumption made by Datlowe and Lin (1973) in advocating the thin-target model on the basis of interplanetary electron spectra.

\section{(iii) Spatial Distribution}

Just how small $\Delta N$ is for the thin-target model depends on the height of the primary electron source. Brown and McClymont (1974) have considered the height distribution of thin target emission above this level, pointing out that it depends only on the density distribution of the target atmosphere. They conclude that for a thin-target $\mathrm{X}$-ray source to be visible in behind-the-limb flares it must be high above the transition layer where $\Delta N \leqslant 10^{18} \mathrm{~cm}^{-2}$ implying efficiencies for the model (compared to thick target emission) of only $3 \%$ at $5 \mathrm{keV}, 0.1 \%$ at $25 \mathrm{keV}$ and only $6 \times 10^{-30} \%$ at $100 \mathrm{keV}$. Hence the total energy and number of electrons needed for a burst are more than 30 times those required by thick target emission and so unacceptably high (cf. Section VI). If on the other hand the source lies below the transition region then $\Delta N$ may be 
$\gtrsim 10^{19} \mathrm{~cm}^{-2}$ but with the source mostly lying only some $2000 \mathrm{~km}$ above the photosphere and not visible in behind-the-limb flares (just as for a chromospheric thick target model). Furthermore, not only does a thin-target require more electron energy in general than a thick-target (and perhaps more than available from a whole active region) but almost all of this energy is 'wasted' by being dumped in the low density corona and making no further contribution to the flare. Brown and McClymont (1974) have emphasised the problems of such dumping by quantitative consideration of the required trap parameters. In particular they conclude that, in a large event, more than $10^{40}$ electrons of $\geqslant 25 \mathrm{keV}$ would have to be trapped extremely high in the corona where $n \lesssim 10^{7} \mathrm{~cm}^{-3}$. Such trapping would produce a vast microwave event unless the trapping field were exceptionally small, requiring in turn a large trapping volume conservatively set at a cubic solar radius.

Finally, it has been widely recognized (e.g. Kane, 1973; Brown, 1973b; Brown and McClymont, 1974) that a purely thin-target model provides no explanation of the synchronism of hard X-rays and EUV bursts from the chromosphere.

\section{(c) THE ELECTRON TRAP MODEL}

Based on the original proposal by Takakura and Kai (1966), this model describes bursts in terms of the bremsstrahlung of electrons magnetically trapped in the low corona. Initially it was supposed that rapid ('impulsive') injection of electrons explained the relatively short rising phase of bursts while the gradual collisional decay of the trapped electrons produced the burst decay profile. More recent observations, however, and particularly their complex temporal fine structure, have required considerable modification of the model.

\section{(i) Time Profiles}

Small bursts which apparently comprise a single spike might be direct examples of the simple impulsive injection/collisional decay hypothesis, but it has been pointed out that the observed softening spectral decay of these spikes conflicts with the hardening expected from collisions (Kane and Anderson (1970) and others). Brown (1972a) has, however, shown that this discrepancy can be resolved if the higher energy electrons encounter a higher mean plasma density along their paths. De Feiter (1974) has criticised this as 'artificial' but in fact the necessary decrease of electron pitch angle with increasing energy is a natural result of direct electric field acceleration (cf. Speiser, 1965; Petrosian, 1973) while some increase of plasma density down the arms of the trap is inevitable (cf. Benz and Gold, 1971). To that extent the explanation of impulsive spike characteristics in a trap model is more satisfactory than in continuous injection models since based on definitive physical features of the model rather than being an ad hoc requirement of the acceleration process.

The complex fine structure of larger events (Section III(a)) can be reconciled with an electron trap source in two ways. Firstly by a mixture of models (Kane, 1974a) in which the acceleration of electrons in the trap is repeated or continuous through the event but with a fraction of the electrons escaping downward (to a thick target) to 
produce the rapid burst fine structure and synchronised chromospheric EUV variations. Secondly, the rapid acceleration hypothesis may be maintained and the burst time structure attributed to MHD oscillations of the trap itself (Parks and Winckler, 1971; Brown, 1973c). This version of the model differs greatly, however, from the original impulsive acceleration concept since the magnetic field variations accompanying the required plasma density oscillations result in induced electric field acceleration of the trapped electrons throughout the event (Brown, 1973c; Brown and Hoyng, 1975).

\section{(ii) Polarisation and Directivity}

No adequate analysis of these features of a trapped electron source has yet been published. Earlier descriptions (Ohki, 1969; Pinter, 1969; Shaw, 1972; Elwert and Haug, 1970, 1971; Tindo et al., 1972b) have considered either electrons moving along a horizontal field or circling horizontally near the trap ends (cf. Brown, 1972a; Benz and Gold, 1971; Haug, 1972), entirely neglecting the magnetic field curvature which is an essential feature of the model. Preliminary results of the detailed calculations necessary to include the field structure (McClymont and Brown, 1974) tend to suggest that both the polarisation and directivity are smaller than earlier predictions and than the raw observational results of Tindo et al. Results for both the degree and planeof polarisation are, however, much more complicated than previously supposed and their evaluation in terms of observations must await complete results of this analysis.

\section{(iii) Electron Acceleration Spectrum}

Since collisional distortion of the electron spectrum occurs only over the whole burst time scale, the instantaneous electron spectrum inferred from Equation (5) at the burst peak can be identified with the electron acceleration spectrum, provided correction is made for the higher plasma densities possibly encountered by higher energy electrons - Brown (1972a) (this correction may amount to over 2 powers steepening of the inferred electron spectrum and so greatly affect estimates of total electron numbers and energy in the model). When the entire evolution of the burst spectrum is considered, however, the time-integrated X-ray spectrum will correspond to that of a thick-target source instantaneously (with the same initial electron injection spectrum) since the collisional spectral evolution is just that within a thick target but on a long time scale.

\section{(iv) Spatial Distribution of Emission}

Quantitative predictions of the spatial distribution of emission from trapped electrons are not yet complete, though under way (McClymont and Brown, 1974). It is clear of course that at least some of the emission will emanate from high in the atmosphere, compatible with behind-the-limb events but there is in fact a considerable concentration of the emission deep in the two limbs of the trap due to the higher density there (Brown, 1972a; Haug, 1972; Benz and Gold, 1971; McClymont and Brown, 1974) and also to the greater path lengths of spiralling source electrons near their reflection points (McClymont and Brown, 1974). 


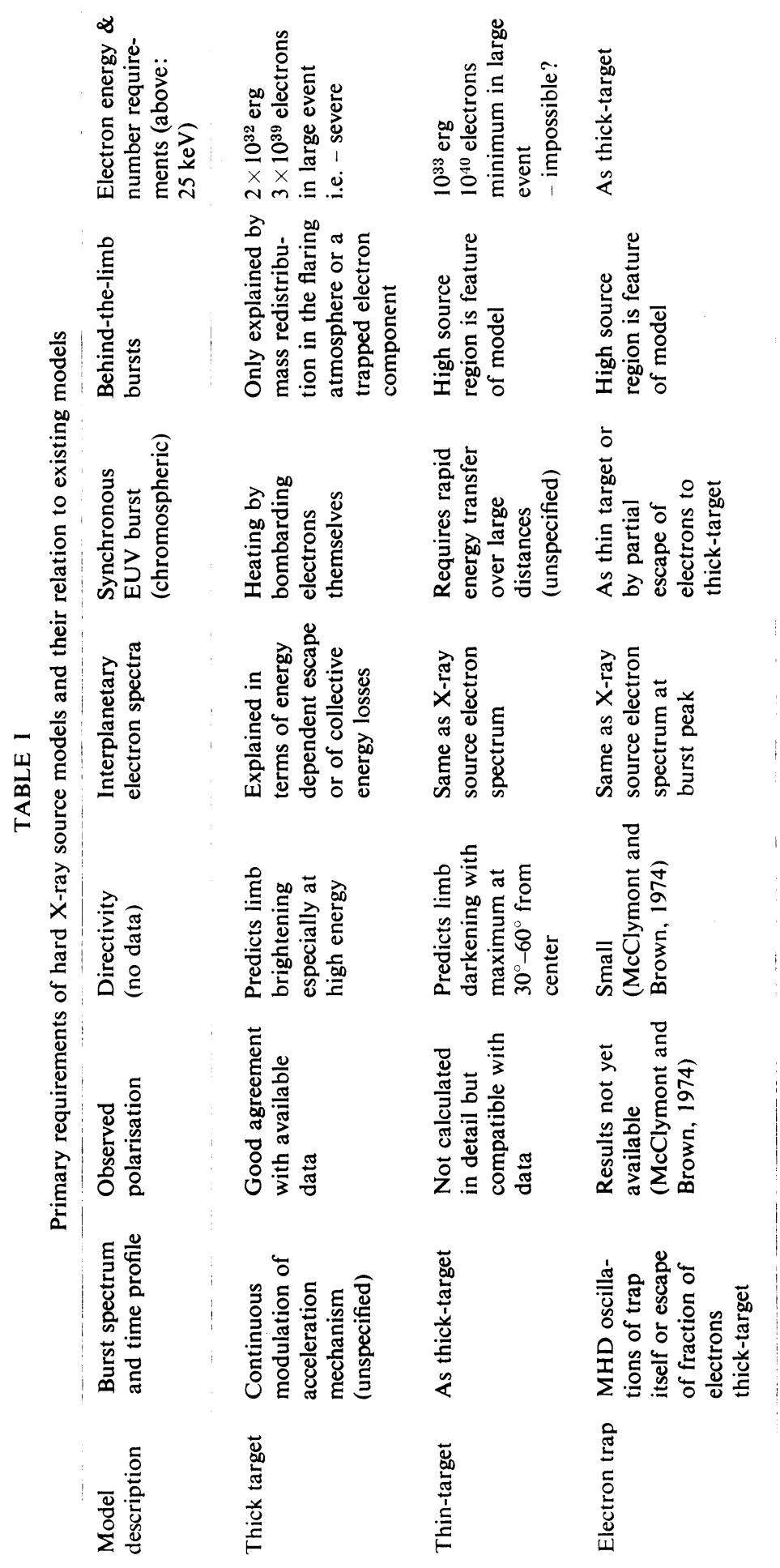


(d) CONCLUSIONS

In Table I are summarised the key requirements of burst models and the abilities of the three above source models to meet these. The essence of the present situation is that the thick-target model may be capable with moderate economy of flare energy, of explaining all burst features including behind-the-limb occurrences, when mass motion of the target flare plasma is important, and interplanetary electron spectra if the electron escape is suitably energy dependent or if collective losses are involved. The thin target, on the other hand, provides an immediate interpretation of interplanetary electron spectra but only explains behind the limb events at the expense of demanding unreasonably large electron fluxes, and without explaining the EUV observations. Finally, coronally trapped electrons are readily compatible with behindthe limb emission and interplanetary electron spectra but may produce too small a polarisation and are hard to reconcile with chromospheric flare emissions unless a substantial (thick-target) downward escape of electrons also occurs.

\section{The Electron Energy and Number Problem}

\section{(a) INTRODUCTION}

As discussed in previous sections, a thick-target is the most efficient of existing source models in terms of the electron flux required to produce any given X-ray burst intensity. As noted in the introduction, however, in some flares even the thick-target model needs a total energy of electrons comparable to or exceeding the total thermal energy. This conclusion was first reached by extrapolating the observed steep power-law spectra below the non-thermal energy range directly observable (Neupert, 1968; Brown, 1971; Kahler and Kreplin, 1971) but the result is now known sometimes to hold without any extrapolation of the X-ray power-law beyond the observed range (e.g. Syrovatskii and Shmeleva, 1972). Typical figures for a large (3B) event (1972, August 4) are those given by Hoyng et al. (1975), namely $4 \times 10^{39}$ electrons of $E_{0} \geqslant 25 \mathrm{keV}$ with a total energy of $2 \times 10^{32} \mathrm{erg}$ (these requirements increasing tenfold if the spectrum is extended down to even $10 \mathrm{keV}$ ). Although flare heating models based on energetic electrons as the mode of energy transport have achieved some success (see Section I), it is a major theoretical problem to find a mechanism capable of accelerating such a number and energy of electrons on the required time scale. The severity of the energy release problem is well known (e.g. Sweet, 1969): The number problem is clearly seen on noting that the acceleration of even $4 \times 10^{39}$ electrons from coronal material, where $n \lesssim 2 \times 10^{9} \mathrm{~cm}^{-3}$ (Lin, 1973), would require total involvement of $2 \times 10^{30} \mathrm{~cm}^{3}$ of plasma, or in practice involvement of a fraction of particles in an even greater volume, and this at a rate sufficiently fast to account for the burst time profile. It is therefore essential to consider any factor which might radically change our estimates of these figures, the following being two possible candidates.

(b) THE THERMAL HARD X-RAY CONTRIBUTION

Chubb et al. (1966) (1971) has claimed that hard X-ray burst data have never satisfacto- 
rily excluded thermal interpretation. The subsequent development of this view and the debate over it have been reviewed elsewhere in this Symposium (Kahler, 1975). Brown (1974) has emphasised that, although thermal emission is hardly capable of explaining $\mathrm{X}$-ray bursts throughout the hard X-ray energy range, it is essential to the problem of non-thermal electron energy in flares to determine the photon energies up to which thermal emission is important. This separation cannot be done on the basis of spectra alone since, as suggested by Chubb (1971) and proved quantitatively by Brown (1974), any hard X-ray spectrum can be produced by a suitable temperature distribution in a thermal plasma. In fact to produce a burst spectrum $I(\varepsilon) \mathrm{cm}^{-2} \mathrm{~s}^{-1} \mathrm{keV}^{-1}$ the emission measure distribution $\mu(T)$, differential in temperature $T(\mathrm{~K})$ should be

$$
\mu(T)\left(\mathrm{cm}^{-3} \mathrm{~K}^{-1}\right)=\frac{1.4 \times 10^{45}}{T^{3 / 2}} \mathscr{L}^{-1}\left\{I(\varepsilon) ; \frac{1.16 \times 10^{7}}{T}\right\},
$$

where $\mathscr{L}^{-1}$ is the inverse Laplace transform (Brown, 1974). In particular to produce power-law spectrum (8), the required distribution is

$$
\mu(T)\left(\mathrm{cm}^{-3} \mathrm{~K}^{-1}\right)=\frac{3.6 \times 10^{34} A}{\Gamma(\gamma-1)}\left[\frac{T}{1.16 \times 10^{7}}\right]^{-\gamma+1 / 2}
$$

while to produce a power-law with high energy break - i.e. $A \varepsilon^{-\gamma} e^{-\varepsilon / \varepsilon_{0}}$

$$
\begin{aligned}
\mu(T) & =\frac{1.4 \times 10^{45} A}{\Gamma(\gamma) T^{3 / 2}}\left[\frac{11.6 \times 10^{6}}{T}-\frac{1}{\varepsilon_{0}}\right]^{\gamma-1} \text { if } T \geqslant 11.6 \times 10^{6} \varepsilon_{0} \\
& =0 \text { otherwise. }
\end{aligned}
$$

(Contrary to De Feiter's (1974) claim, it should be noted that there is no more arbitrariness in this fitting procedure than in the adoption of a power-law electron spectrum for non-thermal models). Against the thermal mechanism Kahler (1971a, b) has claimed firstly that the thermal conduction intrinsic to a distributed temperature source would cool the source too fast to explain bursts and secondly that an electron temperature (i.e. Maxwell distribution) could not be established on the short time scale of rapid burst variations. Brown (1974) has countered that when Equation (24) is used to determine the temperature structure, Kahler's conduction figure is found to be an overestimate, and secondly that if the thermal flare plasma is highly turbulent, conduction is severely inhibited. Furthermore, energy exchange via the turbulent plasmons, greatly reduces the effective Maxwell relaxation time (M. Kuperus and J. Kuipers, 1974, private discussions.) Thus it is the reviewer's opinion that the thermal mechanism for hard X-ray emission still cannot be excluded in the energy range below about $100 \mathrm{keV}$. The important consequence in the present content, however, is that if thermal emission dominated the spectrum of the 1972, August 4 event up to $50 \mathrm{keV}$, the inferred non-thermal electron energy would be only $2 \times 10^{31}$ erg and therefore a quite minor part of the flare. 
Kahler and Kreplin (1971) and Kahler (1973) have attempted to separate thermal and non-thermal emissions by distinguishing the impulsive and gradual components of burst time profiles, and concluded that the non-thermal component can extend right down to a few $\mathrm{keV}$ in some flares. This method suffers from the problems that it does not take account of the dependence of the thermal contribution on the plasma density, the effects of a non-isothermal spectrum, or the possible impulsive profile of transient thermal emission itself. Though theoretical development of Kahler and Kreplin's work along these lines may help clarify the problem, no satisfactory answer may be forthcoming until spatially and spectrally resolved hard X-ray observations and also improved polarisation data over a wide energy range, are obtained. At present polarisation data support the view that thermal flare emission may dominate to well above $15 \mathrm{keV}$ in energy (cf. Frost, 1974a; Brown et al., 1974).

\section{(c) ELECTRON REACCELERATION}

The electron numbers quoted in Section VI(a) and previous sections, and throughout the literature, are based entirely on 'injection' models in which each electron is accelerated once only and subsequently injected into a source region where it (collisionally) emits bremsstrahlung and loses its energy once and for all. If, however, electrons could be accelerated and emit their bremsstrahlung during rapid deceleration, in one and the same region, then they would be available for in situ reacceleration within the source. Two mechanisms potentially capable of combining confinement and repeated acceleration are acceleration by reflection between moving magnetic mirrors (Fermi acceleration) and stochastic acceleration by collisions with plasmons in a turbulent plasma (e.g. Tsytovich, 1973). As shown in Section V(b) the generation of plasma turbulence by particle streams in a thick-target situation has the effect of reducing the electron lifetime and so the bremsstrahlung emission during one 'stopping'. If, however, an electron is contained in the acceleration region, then it may be reaccelerated repeatedly. In this case the total number of electrons obtained by integrating Equation (16) over the event duration is not of physical interest and the total number of electrons required for production of a burst depends instead on the frequency with which an average electron is reaccelerated. The much smaller number of electrons needed could then obviate the severe problem of total electron numbers stated in VI(a) and might reduce the total electron numbers required for a hard X-ray burst to the same order of magnitude as those needed to supply microwave bursts and interplanetary electrons.

The effect of such a reacceleration model on the total electron energy requirements for hard X-ray bursts is less clear. If all the energy lost by an electron in each acceleration cycle is irreversibly lost to heating the ambient plasma, the total energy which passes through the form of energetic electrons must turn out to be as before - i.e. $\gtrsim 2 \times 10^{32}$ erg in a large flare. Even this situation is quite distinct from that implied in 'injection' models, however, since a relatively small number of electrons play a continuous role in the transfer of stored flare energy into plasma heating, but no such total energy need be released into the final form of non-thermal particles. Secondly, it 
would be most important to establish whether the process of rapid energy exchange between plasmons and electrons in this situation may affect the nett energy loss rate of an electron, occurring in Equation (13) (with 'recycling' of energy between electrons and plasma) and so possibly even enhance the efficiency of the source relative to a thick-target injection model, in terms of energy.

Finally it must be recognized that though this line of thought may be worth pursuing from the point of view of efficiency, it is not clear how either an in situ reacceleration model, or a thermal model, could explain the relationship of hard X-ray bursts to chromospheric (EUV) flare emissions or the observed degree of burst polarisation, involving as they do relatively randomised electron velocities and a confined energy release region.

\section{Acknowledgements}

The author wishes to gratefully acknowledge the support of ESRO and of the Dutch National Committee for Geophysics and Space Research during preparation of this review and the provision of funds from the University of Utrecht and from the IAU for attendance of this Symposium.

Discussions of aspects of the review with P. Hoyng, I. Craig, A. McClymont and M. Kuperus and the provision of preprint material by K. Frost, S. Kane, A. Korchak, and $\mathrm{A}$. McClymont were of invaluable assistance.

\section{References}

Acton, L. W.: 1968, Astrophys. J. 152, 305.

Arnoldy, R. L., Kane, S. R., and Winckler, J. R. : 1968, Astrophys. J. 151, 711.

Van Beek, H. F., De Feiter, L. D., and De Jager, C.: 1974, to appear in Proc. Eslab Symp. (Saulgau 1973).

Beigman, I. L. and Vainshtein, L. A.: 1974, Soviet Astron. - A.J. (in press).

Benz, A. O. and Gold, T.: 1971, Solar Phys. 21, 157.

Brown, J. C.: 1971, Solar Phys. 18, 489.

Brown, J. C.: 1972a, Solar Phys. 25, 158.

Brown, J. C.: 1972b, Solar Phys. 26, 441.

Brown, J. C.: 1973a, Solar Phys. 31, 143.

Brown, J. C.: 1973b, Proc. Leningrad Symp. on Solar Cosmic Rays, June 1973.

Brown, J. C.: 1973c, Solar Phys. 32, 227.

Brown, J. C.: 1973d, Solar Phys. 28, 151.

Brown, J. C.: 1974, in G. Newkirk, Jr. (ed.), 'Coronal Disturbances', IAU Symp. 57, 395.

Brown, J. C. and Hoyng, P.: 1975, submitted to Astrophys. J.

Brown, J. C., McClymont, A. N., and McLean, I. S.: 1974, Nature 247, 448.

Brown, J. C. and McClymont, A. N.: 1974, Solar Phys. 41, 135.

Canfield, R. C.: 1974, Solar Phys. 34, 339.

Cheng, C. C.: 1972, Solar Phys. 22, 178.

Chubb, T. A.: 1971, in E. R. Dyer (General Ed.), Solar-Terrestrial Physics/1970, Part 1, D. Reidel Publ. Co., Dordrecht, Holland, p. 99.

Chubb, T. A., Kreplin, R. W., and Friedman, H.: 1966, J. Geophys. Res. 71, 3611.

Cline, T. L., Holt, S. S., and Hones, E. W.: 1969, J. Geophys. Res. 73, 434.

Craig, I. J. D.: 1974, Ph.D. Thesis, University College, London.

Craig, I. J. D. and Brown, J. C. : 1974, unpublished work.

Datlowe, D. W.: 1975, This volume, p. 191. 
Datlowe, D. W. and Lin, R. P.: 1973, Solar Phys. 32, 459.

Dere, K. P., Horan, D. M., and Kreplin, R. W.: 1974, Solar Phys. in press.

Drake, J. F.: 1971, Solar Phys. 16, 152.

Elwert, G.: 1968, in K. O. Kiepenheuer (ed.), 'Structure and Development of Solar Active Regions', IAU Symp. 35, 144.

Elwert, G. and Haug, E.: 1970, Solar Phys. 15, 234.

Elwert, G. and Haug, E.: 1971, Solar Phys. 20, 413.

De Feiter, L. D.: 1974, Space Sci. Rev. 16, 3.

Fox, L. and Mayers, D. F.: 1968, Computing Methods for Scientists and Engineers, Clarendon Press, Oxford.

Frost, K. J.: 1969, Astrophys. J. 158, L159.

Frost, K. J.: 1974a, in G. Newkirk, Jr. (ed.), 'Coronal Disturbances', IAU Symp. 57, 421.

Frost, K. J.: 1974b, Private communication.

Frost, K. J. and Dennis, B. R.: 1971, Astrophys. J. 165, 655.

Haug, E.: 1972, Solar Phys. 25, 425.

Herring, J. R. H. and Craig, I. J. D.: 1973, Solar Phys. 28, 169.

Householder, A. S.: 1964, The Theory of Matrices in Numerical Analysis, Blaisdell Pub. Co.

Hoyng, P., Brown, J. C., Stevens, G. A., and Van Beek, H. F.: 1975, This volume p. 233.

Hoyng, P. and Stevens, G. A.: 1974, Astrophys. Space Sci. 27, 307.

Hudson, H. S.: 1972, Solar Phys. 24, 414.

Hudson, H. S.: 1973, in R. Ramaty and R. G. Stone (eds.), High Energy Phenomena on the Sun, NASA SP-342, p. 207.

De Jager, C. and Kundu, M. R.: 1963, Space Res. 3, 836.

Kahler, S. W.: 1971a, Astrophys. J. 164, 365.

Kahler, S. W.: 1971b, Astrophys. J. 168, 319.

Kahler, S. W.: 1973, in R. Ramaty and R. G. Stone (eds.), High Energy Phenomena on the Sun, NASA SP-342, p. 124.

Kahler, S. W.: 1975, This volume, p. 211.

Kahler, S. W. and Kreplin, R. W.: 1971, Astrophys. J. 168, 531.

Kane, S. R.: 1969, Astrophys. J. 157, L139.

Kane, S. R.: 1971, Astrophys. J. 170, 587.

Kane, S. R.: 1973, in R. Ramaty and R. G. Stone (eds.), High Energy Phenomena on the Sun, NASA SP-342, p. 55.

Kane, S. R.: 1974a, in G. Newkirk, Jr. (ed.), 'Coronal Disturbances', IAU Symp. 57, 105.

Kane, S. R.: 1974b, paper presented at AAS meeting, University of Hawaii, Jan. 1974.

Kane, S. R. and Anderson, K. A.: 1970, Astrophys. J. 162, 1003.

Kane, S. R. and Donnelly, R. F.: 1971, Astrophys. J. 164, 151.

Kawabata, K., Sofue, Y., Ogawa, H., and Omodaka, T.: 1973, Solar Phys. 31, 469.

Koch, H. W. and Motz, J. W.: 1959, Rev. Mod. Phys. 31, 920.

Korchak, A. A.: 1967a, Soviet Astron. - A.J. 11, 258.

Korchak, A. A.: 1967b, Soviet Phys. Doklady 12, 192.

Korchak, A. A.: 1971, Solar Phys. 18, 284.

Korchak, A. A.: 1974, preprint.

Kuperus, M. and Kuipers, J.: 1974, Private communication.

Lin, R. P.: 1974a, Space Sci. Rev. 16, 184.

Lin, R. P.: 1974b, in G. Newkirk, Jr. (ed.), 'Coronal Disturbances', IAU Symp. 57, 201.

McClymont, A. N. and Brown, J. C.: 1974, Solar Phys. in press.

McKenzie, D. L., Datlowe, D. W., and Peterson, L. E.: 1973, Solar Phys. 28, 175.

Neupert, W. M.: 1968, Astrophys. J. 153, L59.

Ohki, K.: 1969, Solar Phys. 7, 260.

Parks, G. R. and Winckler, J. R.: 1969, Astrophys. J. 155, L117.

Parks, G. R. and Winckler, J. R.: 1971, Solar Phys. 16, 186.

Peterson, L. E., Datlowe, D. W., and McKenzie, D. L.: 1973, in R. Ramaty and R. G. Stone (eds.), High Energy Phenomena on the Sun, NASA SP-342, p. 132.

Petrosian, V.: 1973, Astrophys. J. 186, 291.

Pinter, S.: 1969, Solar Phys. 8, 142.

Santangelo, N., Horstman, H., and Horstman-Moretti. E.: 1973, Solar Phys. 29. 143. 
Schatzmann, E.: 1966, in C. de Jager (ed.), The Solar Spectrum, D. Reidel Publ. Co., Dordrecht, Holland, p. 313.

Shaw, M. L.: 1972, Solar Phys. 27, 436.

Shmeleva, O. P. and Syrovatskii, S. I.: 1973, Solar Phys. 33, 341.

Smith, D. F.: 1974, in G. Newkirk, Jr. (ed.), 'Coronal Disturbances', IAU Symp. 57, 253.

Speiser, T. W.: 1965, J. Geophys. Res. 20, 4219.

Strauss, F. M. and Papaggianis, M. D.: 1971, Astrophys. J. 164, 369.

Sturrock, P. A. and Coppi, B. A.: 1966, Astrophys. J. 143, 3.

Sweet, P. A.: 1969, Ann. Rev. Astron. Astrophys. 7, 149.

Sweet, P. A.: 1971, in R. Howard (ed.), 'Solar Magnetic Fields', IAU Symp. 43, 457.

Syrovatskii, S. I. and Shmeleva, O. P.: 1972, Soviet Astron. - A.J. 16, 273.

Takakura, T.: 1969, Solar Phys. 6, 133.

Takakura, T.: 1973, in R. Ramaty and R. G. Stone (eds.), High Energy Phenomena on the Sun, NASA SP-342, p. 179.

Takakura, T.: 1975, This volume, p. 299.

Takakura, T. and Kai, K.: 1966, Publ. Astron. Soc. Japan 18, 57.

Takakura, T., Ohki, K., Shibuya, N., Fujii, M., Matsuoka, M., Miyamoto, S., Nishimura, J., Oda, M., Ogawara, Y., and Ota, S.: 1971, Solar Phys. 16, 454.

Thomas, R.: 1975, This volume, p. 25.

Tindo, I. P., Ivanov, V. D., Mandel'shtam, S. L., and Shuryghin, A. I.: 1970, Solar Phys. $14,204$. Tindo, I. P., Ivanov, V. D., Mandel'shtam, S. L., and Shuryghin, A. I. : 1972a, Solar Phys. $24,429$.

Tindo, I. P., Ivanov, V. D., Valnicek, B., and Livshits, M. A.: 1972b, Solar Phys. $27,426$.

Tindo, I. P., Mandel'shtam, S. L., and Shuryghin, A. I.: 1973, Solar Phys. 32, 469.

Tomblin, F. F.: 1972, Astrophys. J. 171, 377.

Tsytovich, V. N.: 1973, Ann. Rev. Astron. Astrophys. 11, 363.

Vorpahl, J. A.: 1973, Solar Phys. 28, 115.

Vorpahl, J. A. and Takakura, T.: 1974, Astrophys. J. (in press).

Wild, J. P. and Smerd, S. F.: 1972, Ann. Rev. Astron. Astrophys. 10, 159.

Wolff, R. S. : 1973, in R. Ramaty and R. G. Stone (eds.), High Energy Phenomena on the Sun, NASA SP-342, p. 162.

Zirin, H. and Tanaka, T.: 1973, Solar Phys. 32, 173. 